\title{
Transcriptomic and metabolomic joint analysis reveals distinct flavonoid biosynthesis regulation for variegated testa color development in peanut (Arachis hypogaea L.)
}

\section{Mengdie Hu}

Hebei Agricultural University, Baoding, China 071001

Jiawei Li

Hebei Agricultural University, Baoding, China 071001

Mingyu Hou

Hebei Agricultural University, Baoding, China 071001

Xiaoqing Liu

Hebei Agricultural University, Baoding, China 071001

Shunli Cui

Hebei Agricultural University, Baoding, China 071001

\section{Xinlei Yang}

Hebei Agricultural University, Baoding, China 071001

Lifeng Liu

Hebei Agricultural University, Baoding, China 071001

\section{Xiaoxia Jiang}

Hebei Yiyuan Ecological Agriculture Technology Co, Ltd, Baoding, China 074200

Guojun Mu ( $\nabla$ mgj99999@126.com )

Hebei Agricultural University, Baoding, China 071001

\section{Research Article}

Keywords: peanut testa, anthocyanin, flavone biosynthesis metabolic pathway, RNA-seq, qPCR

Posted Date: December 1st, 2020

DOI: https://doi.org/10.21203/rs.3.rs-119186/v1

License: (c) (1) This work is licensed under a Creative Commons Attribution 4.0 International License.

Read Full License 


\title{
Transcriptomic and metabolomic joint analysis reveals distinct
}

\section{flavonoid biosynthesis regulation for variegated testa color development in peanut (Arachis hypogaea L.)}

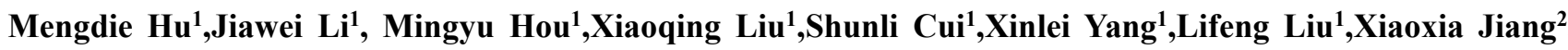 \\ Guojun $\mathrm{Mu}^{1 *}$ \\ 1.North China Key Laboratory for Crop Germplasm Resources of Education Ministry, College of Agronomy, Hebei Agricultural \\ University, Baoding, China 071001. \\ 2.Hebei Yiyuan Ecological Agriculture Technology Co, Ltd, Baoding, China 074200 \\ 304668586@qq.com (M.H.);1796890718@qq.com (J.L.);houmy@hebau.edu.cn (M.H.);3308242@qq.com (X.L.); \\ cuishunli1999@163.com (S.C.);yangxinlei2500@163.com (L.Y.);liulifeng@hebau.edu.cn (L.L.);bdyyny@139.com (X.J.) \\ * Correspondence:mgj99999@126.com;Tel:+86-1380-326-0389
}

\begin{abstract}
Peanut (Arachis hypogaea L.) is one of the important oil and economic crops, among which the variegated testa peanut is a unique member. But the molecular mechanisms underlying the pigment synthesis in variegated testa are still unclear. Differentially expressed genes (DEGs) in pigment metabolism pathway in colored area indicated there were 27 DEGs highly related to the synthesis of variegated testa color among 1,050 DEGs, which were 13 up-regulated and 14 down-regulated,consisting of 3 PALs, 1 C4H, 2 CHSs, 1 F3H, 1 F3' H, 2 DFRs, 2 LARs, 2 IAAs, 4 bHLHs and 9 MYBs. GO analysis indicated DEGs were similarly enriched in 3 branches.KEGG analysis suggested flavonoid biosynthesis is the most direct metabolic pathway for the synthesis of testa variegation.The liquid chromatography tandem mass spectrometry (LC-MS/MS) results showed that cyanidin and delphinidin were the main metabolites that caused the color difference between the colored area and the non-colored area. Through the verification of 20 DEGs via qPCR, the results were consistent with that of transcriptome sequencing in 4 comparison groups. The results in this study lay the foundation for revealing the molecular regulation mechanisms of anthocyanin synthesis in variegated testa peanut.
\end{abstract}

\section{Introduction}

Peanut (Arachis hypogaea L.) is the primary oil and economic crop that occupies an important position in the world for grain, oil, and food. It is widely planted in developing countries in Asia, Africa, and South America, as well as some developed countries including the United States and Australia ${ }^{1}$. Peanut testa contains a variety of biologically active substances, which are important raw materials for cosmetics and medicinal health products ${ }^{2}$. The variegated testa peanut is a unique member of the peanut family. The variegated testa is composed of the colored area and non-colored area, in which the colored area also includes purple, red, pink, and others. Different types and contents of anthocyanins will cause peanut testa to show different colors.Anthocyanins are natural antioxidants ${ }^{3}$, which have a strong affinity for collagen ${ }^{4}$, and have many pharmacological functions in anti-oxidation, anti-inflammatory, eyesight protection, antibacterial, and other aspects ${ }^{5}$.

The development of different colors on the same variegated testa is a very interesting natural phenomenon despite they have the same genetic background. However, the molecular mechanism of pigment synthesis in the testa is still unclear.Plant anthocyanin metabolism is a complex process that included chalcone synthase (CHS), chalcone isomerase (CHI), dihydroflavonnol-3-hydrogenase,(F3H), dihydroflavonol-3'-hydrogenase(F3'H), dihydroflavonol-3' -5 'hydrogena, 
Dioxanonol-4-reductase (DFR), anthocyanidins synthase(ANS), and many other key enzymes ${ }^{6}$.Meanwhile, basic helix-loop-helix (bHLH), v-myb avian myeloblastosis viral oncogene homolog (MYB), WD40 protein, and other transcription factors can also regulate the expression of structural genes in the plant anthocyanin metabolic pathway ${ }^{7}$. The first enzyme (CHS) in anthocyanin synthesis can affect the shade of plant color ${ }^{8}$ and the color of the flower can change from purple to pink or even white if the CHS antisense gene is transformed in Petunia hybrida ${ }^{9}$. The color differentiation of tea tree (Camellia sinensis) buds and leaves are catalyzed by DFR and ANS from three main anthocyanidins ${ }^{10-13}$. MYB and bHLH are important regulatory genes in the anthocyanin synthesis pathway of Petunia hybrida ${ }^{14}$. Overexpression of Rs-MYBI in petunia activates the expression of $A N S$ and DFR in the anthocyanin synthesis process and increases the anthocyanin content ${ }^{15}$. The regulation of anthocyanin synthesis by bHLH transcription factors has been widely investigated in Arabidopsis, snapdragon, chrysanthemum, begonia, and other plants ${ }^{16-18}$. The degree of coloration often only requires the difference in the expression of one or several key anthocyanidin synthesis catalytic enzyme genes between the colored area and the non-spotted area and does not require all the anthocyanidin synthesis catalytic enzyme genes to have an obvious expression difference ${ }^{19}$. For example, the formation of white star-shaped spots in $P$. hybrida is caused by the inhibition of CHS-A expression ${ }^{20}$. The large purple spots in Phalaenopsis stuartiana is primarily due to the specific expression of $D F R$ in the spot area ${ }^{21}$, while others are caused by the expression difference of two or more anthocyanidin synthesis catalytic enzyme genes. For example, the color develops if the expression of $O g C H I$ and $O g D F R$ is suppressed in Oncidium hybridum ${ }^{22}$. Xia et al. found that the up-regulation of DFR in Chinese peanut variety ZH9 is beneficial to the promotion of dihydroflavonol synthesis in the anthocyanin and flavonol synthesis pathways ${ }^{18}$. Li et al. studied four types of peanut testa with different colors 40 and 50 days after anthesis and found that $C H S$ was expressed in large quantities, promoting anthocyanin synthesis. In addition, the expression of $D F R$ was significantly related to the testa color $^{23}$. Wan et al. adopted transcriptome sequencing to analyze the color accumulation of the testa of the pink peanut variety ZH16, showing that the expression of PAL, 4CL, CHS, CHI, and other enzymes increased, while the expression of ANR and LAR decreased ${ }^{24}$. Li et al. found that the relative expression level of $F 3 H$ in purple peanut variety $\mathrm{FH} 01$ is positively correlated with anthocyanin content ${ }^{25}$. One reason for the lack of research on the pigment synthesis and molecular mechanism of the variegated peanut testa is related to the scarcity of the research materials.

Transcriptome sequencing is a powerful technique that can identify almost all transcripts and gene sequences of a specific cell or tissue of a certain species in a certain state and can be used to study gene expression, gene function, structure, alternative splicing, and forecasting new transcripts. Ye et al. adopted this technology to screen out 15 key structural genes involved in the metabolism of peach skin anthocyanins, including the upstream phenyl propionic acid metabolism pathway genes, such as $P A L, C 4 H$, and $4 C L$, and the late anthocyanin metabolism pathway genes, such as $C H S, C H I, F 3 H, F 3^{\prime} H, D F R, L A R$, and $A N R^{26}$. Zhang et al. found that the expression levels of anthocyanin biosynthesis genes $P A L$ and $C 4 H$ and transcription factor RsMYB1 and RsTT8 were significantly down-regulated during the development in a radish variety "Xin Li Mei" ${ }^{27}$. Li et al. concluded that ANS is the key gene determining kiwifruit anthocyanin synthesis via transcriptome sequencing ${ }^{28}$. Jin et al. analyzed transcriptomes of differently colored Cineraria flowers (white and yellow), which were not able to accumulate anthocyanins due to mutations in the coding regions of specific genes that cause variations in color developmen $\mathrm{t}^{29}$. Transcriptome sequencing has been widely used to study various species including apple $\mathrm{e}^{30}$, grape $^{31}$, kiwifruit ${ }^{32}$ and soybean ${ }^{33}$.

In this study, we used transcriptomics to quantitatively analyze the differential genes related to the anthocyanin synthesis pathway between the non-colored area and colored area in the variegated testa of peanut accession VG-01. The relative content of anthocyanins in different parts of the variegated testa at different developmental stages was determined combined with metabonomics to screen out the key genes in the anthocyanin synthesis process of peanut. 
This paper initially revealed the molecular regulation mechanism of anthocyanin synthesis in variegated testa peanut and facilitate further in-depth studies in peanut functional genomics.

\section{Results}

Characterization of variegated testa by stereo microscope. Pigmentation in colored area (F) of the variegated peanut testa began DAF30, which is the active period of related gene expression. The "a" and " $b$ " values of the non-colored area (B) were lower than in colored area during testa development with a slight change, and the " $L$ " value in non-colored area was higher than in colored area, possibly indicative of the white color in non-colored area. As the pod matured after DAF45, the color of variegated testa stabilized. The values of " $a$ " and " $b$ " in colored area changed slightly and "L" increased $12.85 \%$ (Fig. 1, Table S1). The colored areaand the non-colored areaare located in the same seed coat with identical genetic backgrounds, which is ideal for the study of DEGs in variegated testa pigmentation.

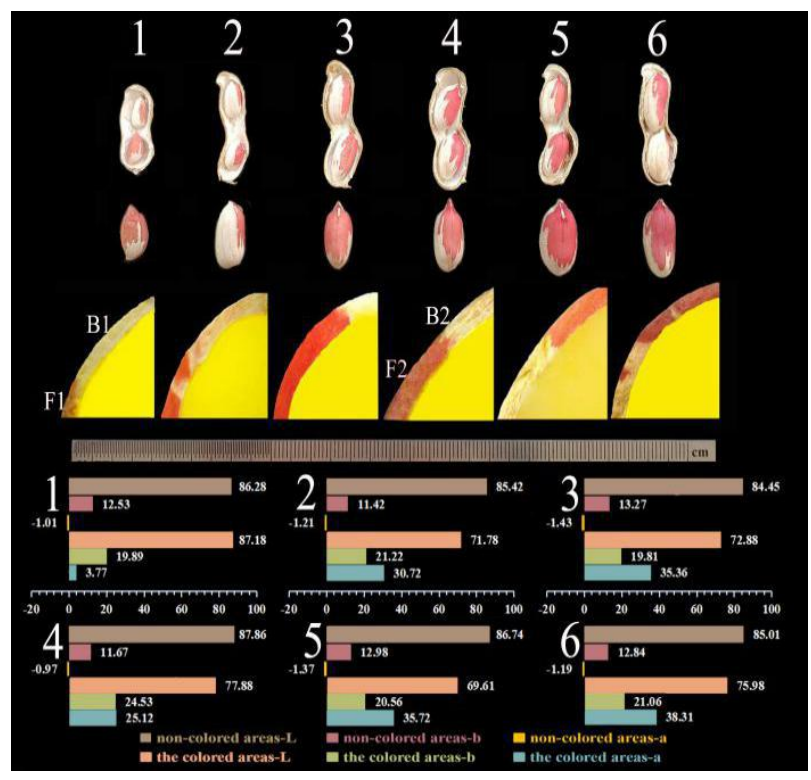

Figure 1. Phenotype and chromatic aberration changes in the color synthesis of variegated peanut testa. 1-6: day after flowering and needling (DAF)30,35,40,45,50, and 55 days. F1 and F2 represent the sample names of colored area at DAF30 and DAF45,respectively. B1 and B2 represent the sample names of non-colored area at DAF30 and DAF45, respectively.The color of the bar represents different values of "a","b","L".The "a" absorbance value ("a" value) represents the red and green. The "b" absorbance value ("b" value) represents yellow and blue and the "L" value represents lightness.

Measurement of anthocyanins. The colored (F1 and F2) and the non-colored (B1 and B2) areas were compared and analyzed horizontally and vertically at DAF 30 and DAF45 In the comparison of F1-B1, the content of procyanidin A1, A2, B2, B3, delphinidin, cyanidin, cyanidin 3-O-galactoside, and rosinidin O-hexoside in colored area increased by 185.40-895.58\%, while the relative content of petunidin 3-O-glucoside and cyanidin O-syringic acid in colored area were lower than in non-colored area, which decreased by 62.70-76.92\% (Fig. 2a). In the comparison of F2-B2, the relative content of procyanidin A1, A2, B2, B3, delphinidin 3-O glucoside, delphinidin, cyanidin, cyanidin 3-O-galactoside, rosinidin O-hexoside in the color areas increased by 106.54-1,759.77\%, while the relative content of cyanidin 3-O-galactoside and cyanidin O-syringic acid in non-colored area increased by 35.25-80.35\%, compared with that in colored area (Fig. 2b). In the comparison of B1-B2, the relative content of petunidin 3-O-glucoside, delphinidin, cyanidin 3-O-galactoside at DAF45 increased by 43.73-81.62\%, while the relative content of cyanidin O-syringic acid, cyanidin 3-O-glucoside, procyanidin A1, rosinidin O-hexoside at DAF45 decreased by 152.39-313.82\% compared with DAF30 
(Fig. 2c). The metabolites between F1 and F2 were almost identical. The relative content of delphinidin 3-O-glucoside, petunidin 3-O-glucoside, and rosinidin O-hexoside at DAF45 was 48.44-92.25\% higher than at DAF30, while the relative content of cyanidin O-syringic acid at DAF45 decreased by $386.24 \%$ (Fig. 2d, Table S2).
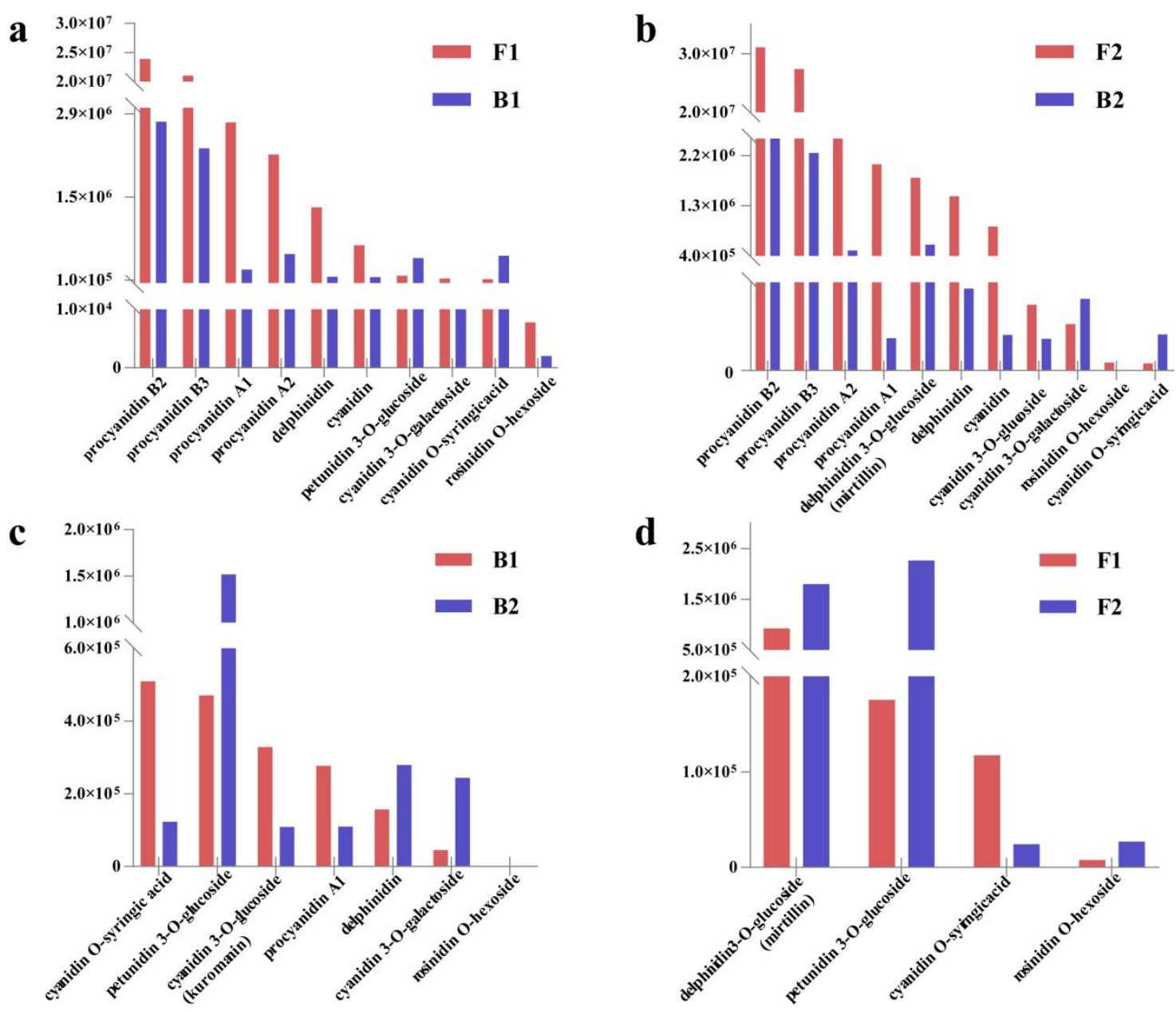

Figure 2. Types and relative content of anthocyanins in different areas of peanut testa at different developmental stages. a and b: represent the type and content differences of metabolized anthocyanins between the colored and non-colored areas after DAF30 and DAF45, respectively. c: Represents the different metabolized anthocyanin types and content in the non-colored area after DAF30 and DAF45. d: Represents the different metabolized anthocyanin types and content in the colored area at DAF30 and DAF45. The horizontal axis represents the types of anthocyanins in the variegated testa and the vertical axis represents the peak area value determined by the LC-MS/MS detection platform.

RNA Sequencing. Transcriptome sequencing was performed on F1, B1, F2, and B2 of the variegated peanut accession VG-01. The contaminated adapter sequences and low-quality read sequences were all removed from the sequencing data. A total of $64.33 \mathrm{~Gb}$ clean data were obtained, and the clean data of each sample reached $6.53 \mathrm{~Gb}$. The base recognition error rate was about $0.02 \%$, the Q20 value of the obtained sequence was above $97.45 \%$, the Q30 base percentage was above $93.23 \%$, and the GC content was above $43.90 \%$ (Table S3)

Detection of DEGs. Comparison to the cultivar tetraploid reference genome of peanut and hierarchical clustering analysis based on the FPKM of the DEGs, a total of 214 differential genes in F1-B1 were identified, of which 53 were up-regulated and 161 were down-regulated. There were 348 differential genes identified in F2-B2, including 97 up-regulated and 251 down-regulated. There was a total of 152 differential genes in B1-B2, including 82 up-regulated 
and 70 down-regulated genes, and 213 differential genes in F1-F2, with 169 up-regulated and 44 down-regulated (Fig. 3 , Table S4).
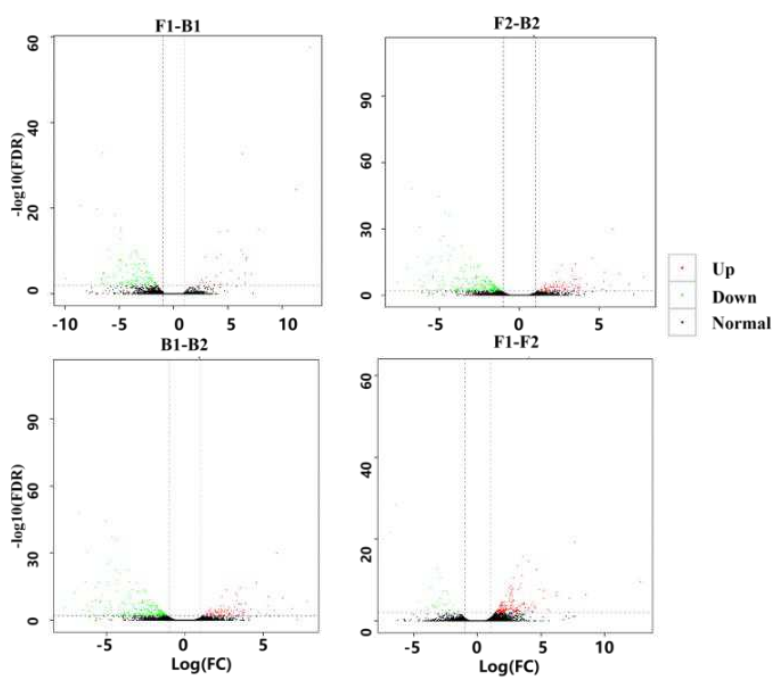

Figure 3. Analysis of the volcano map of different genes in various parts of the peanut testa at different developmental stages. The abscissa indicates the multiple of difference, and the ordinate indicates -log10 (q-value). Red dots indicate up-regulation of gene expression, green dots indicate down-regulation of gene expression, and black dots indicate that there is no significant difference in gene expression.

GO enrichment analysis of DEGs. 1,050 DEGs (accounting for $82.48 \%$ of all differential genes) were compared with GO public databases of tetraploid cultivated peanut and diploid wild peanut, respectively. The comparison results of the tetraploid reference genome showed that F1-B1, F2-B2, F1-F2, and B1-B2 comparison groups were all enriched in 51 GO entries and the diploid genome comparison showed that 4 comparison groups were all enriched in 41 GO entries (Table S5). Following comparative analysis, the enrichment items in 3 branches of 4 comparison groups were horizontally similar. Analysis of the tetraploid cultivar transcriptome data revealed that the enriched items in the cellular components category were membrane, and membrane part, the molecular function were enriched in catalytic activity, binding, and transporter activity. In addition, biological processes showed that DEGs enriched in metabolic, single-organism, and cellular processes (Fig.4). 


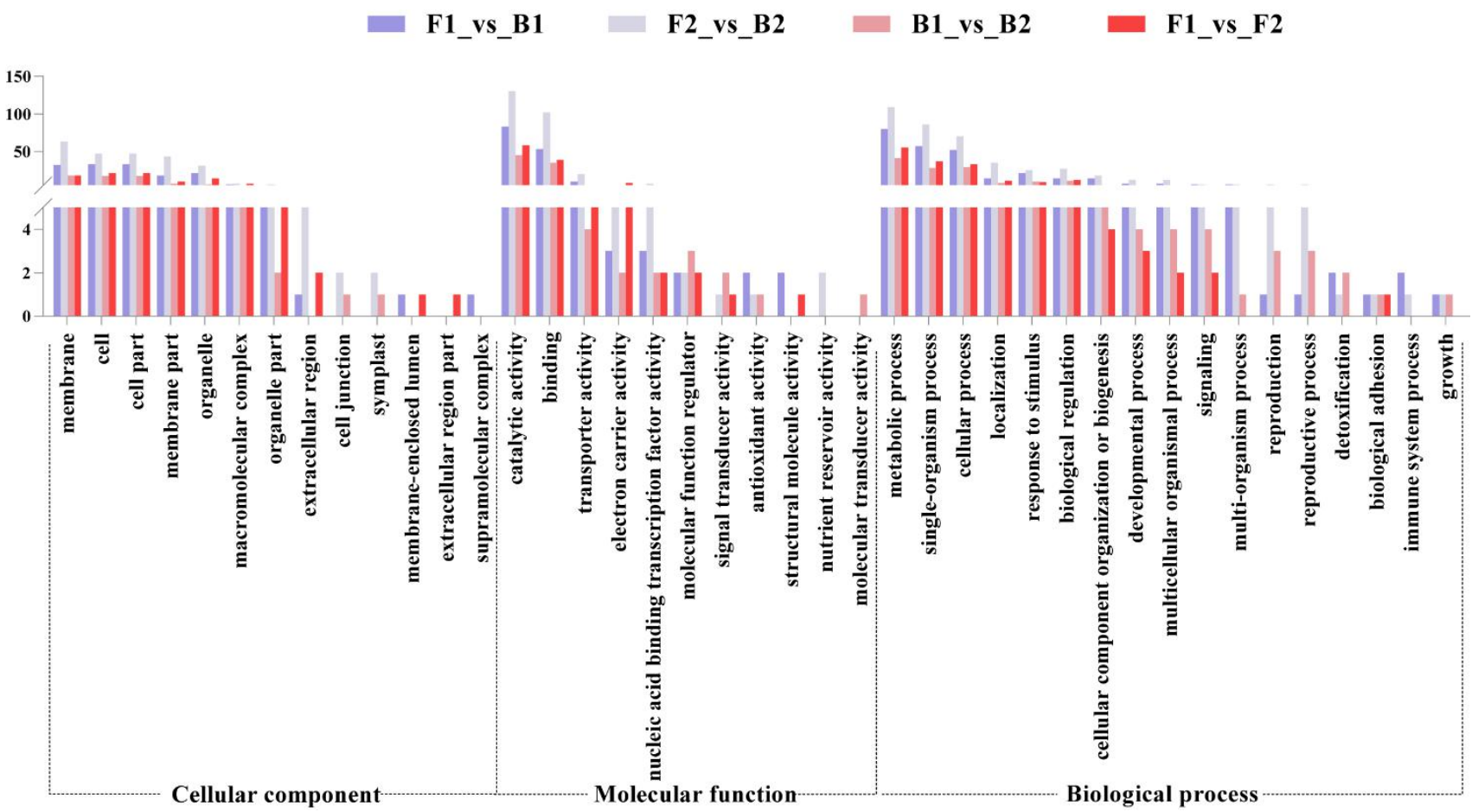

Figure 4. GO functional enrichment classification of variegated peanut testa transcriptome .Different colors bars represent 4 comparison groups, respectively. Y-axis represents number of genes mapped to indicated GO term, and x-axis represents each GO term.GO terms with an adjusted $\mathrm{p}$-value of $<0.05$ were considered signifificantly enriched.

COG enrichment analysis of DEGs. The annotated genes were compared with the COG database and it was found that F1-B1 contained the largest DEGs proportion (R-General functional prediction only), accounting for 12.61\%, followed by Transcription, accounting for $6.54 \%$. Amino acid transport and metabolism accounted for $5.61 \%$. Energy production and conversion, and Defense mechanisms accounted for 4.67\%. F2-B2 had the largest DEGs proportion (R-General functional prediction only), accounting for $10.63 \%$, followed by Transcription, accounting for $6.32 \%$. Amino acid transport and metabolism accounted for $5.61 \%$. Energy production and conversion, and Defense mechanisms accounted for 4.89\%. DEGs in B1-B2 contained the largest DEGs proportion (R-General functional prediction only), accounting for $5.92 \%$, followed by Transcription, accounting for $4.61 \%$. The synthesis, transport, and metabolism of Secondary metabolites biosynthesis accounted for 3.29\%. F1-F2 had the largest DEGs proportion (R-General functional prediction only), accounting for $7.51 \%$, followed by Modification and transport after transcription and protein translation, accounting for 5.63\% (Fig. 5). 


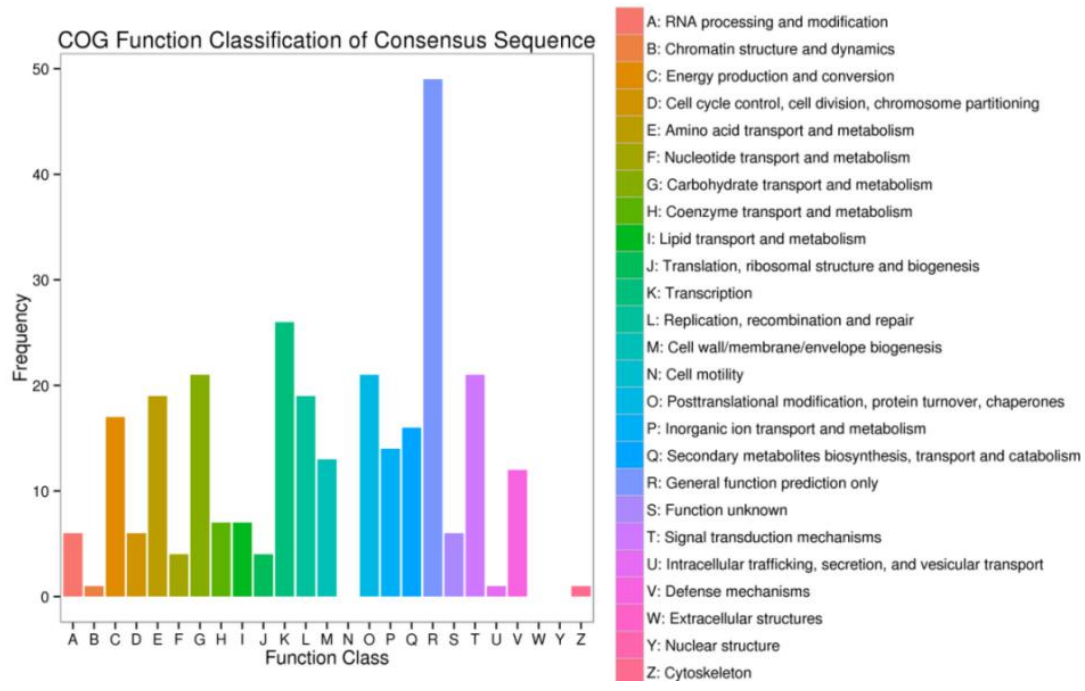

Figure 5. COG functional classification compared with the tetraploid peanut reference genome. A: RNA processing and modification; B: Chromatin structure and dynamics; C: Energy production and conversion; D: Cell cycle control, cell division, chromosome partitioning: E: Amino acid transport and metabolism; F; Nucleotide transport and metabolism; G: Carbohydrate transport and metabolism; H: Coenzyme transport and metabolism; I; Lipid transport and metabolism; J: Translation, ribosomal structure and biogenesis; K: Transcription; L: Replication, recombination and repair: M: Cell wall/membrane/envelope biogenesis; N: Cell motility; O: Posttranslational modification protein turnover, chaperones; P: Inorganic ions transport and metabolism; Q: Secondary metabolites biosynthesis, transport and catabolism; R: General functional prediction only; S: Function unknown; T: Signal transduction mechanism; U: Intracellular trafficking, secretion, and vesicular transport; V: Defense mechanisms; W: Extracellular structures; Y: nuclear structure; Z: Cytoskeleton.

KEGG pathways enrichment analysis of DEGs. In order to understand the biological functions of DEGs discussed above, the transcriptome sequencing results were compared with the KEGG public database of tetraploid cultivated peanut and diploid wild peanut. The results showed that 363 DEGs $(28.52 \%$ of all differential genes) were annotated in the KEGG database. Among them, the metabolic pathways associated with testa color enrichment in F1-B1 included phenylalanine metabolism, phenylpropanol biosynthesis, flavonoid biosynthesis, and plant circadian rhythm. Testa color-related enrichment metabolic pathways in F2-B2 included phenylpropanol biosynthesis, flavonoids and flavonol biosynthesis, and flavonoid biosynthesis. Testa color-related enrichment metabolic pathways in B1-B2 included plant hormone signals transduction and biosynthesis of phenylalanine, tyrosine and tryptophan. Testa color-related enrichment pathways in F1-F2 included phenylalanine, tyrosine, and tryptophan biosynthesis. The diploid and tetraploid reference genomes were compared based on different parts of the same period and different periods of the same part and 6 metabolic pathways related to anthocyanin biosynthesis were screened out, including phenylpropane acid metabolism, phenylpropanol biosynthesis, flavonoids and flavonol biosynthesis, flavonoid biosynthesis, plant hormone signal transduction, and circadian rhythm in plants. (Fig. 6, Table S6). 


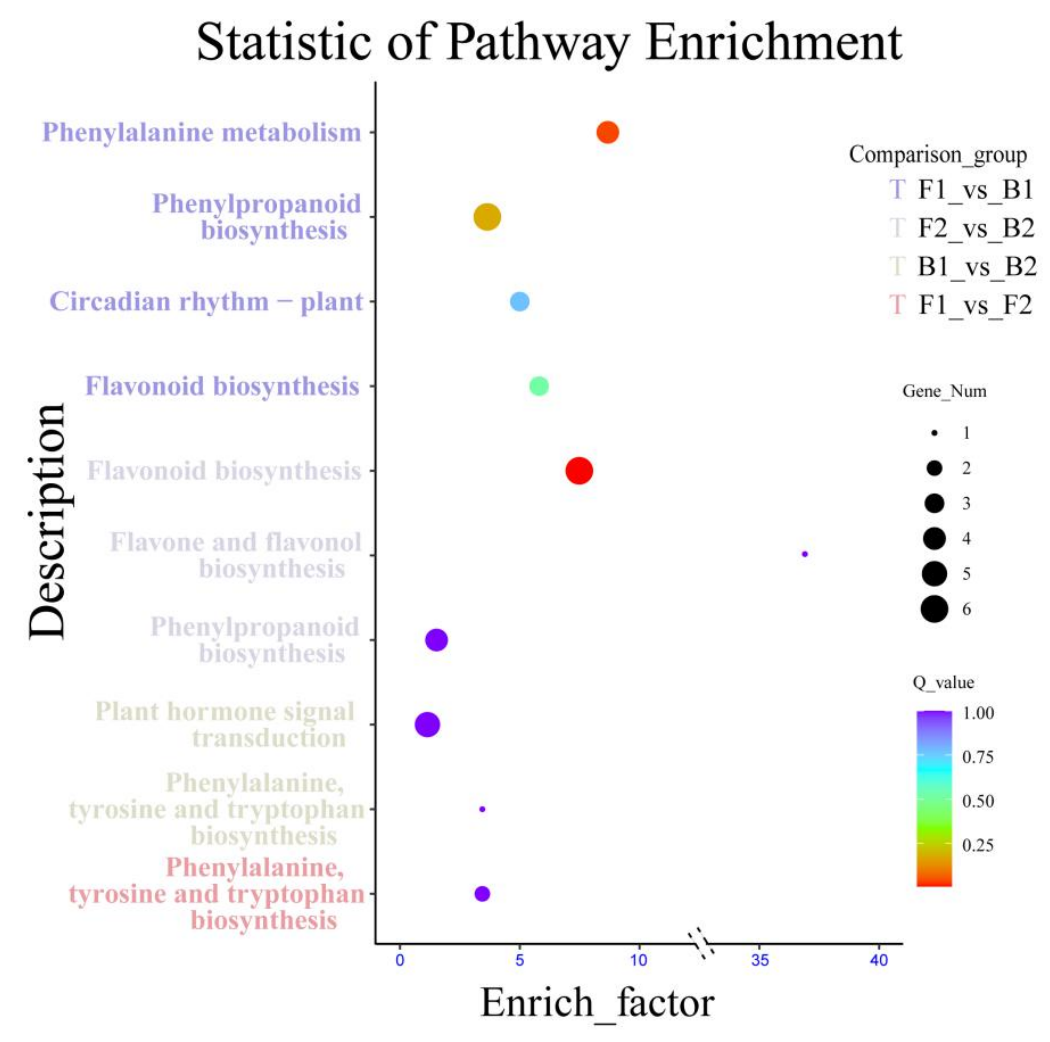

Figure 6. Pathway enrichment analysis of DEGs. The ordinate represents the pathway entry, and the abscissa represents the rich factor. The dots represent the number of significantly DEGs, and the significance increases as the dots increase in size. The dots with different colors indicate different Q-values.

Structural genes related to testa pigment synthesis. The Veen diagram analysis showed that 71 DEGs co-expressed between F1-B1 and F2-B2, and 143 DEGs presented specifically F1-B1 while 277 DEGs particularly expressed in F2-B2(Fig.7c, Table S7). 15 DEGs co-expressed between B1-B2 and F1-F2, and 137 DEGs presented specifically B1-B2 while 198 DEGs particularly expressed in F1-F2 (Fig.7c, Table S7). KEGG analysis showed that both F1-B1 and F2-B2 involved differential genes related to peanut testa color and the expression of DEGs was closely related to testa development. Genes involved in the pigment synthesis included 3 PAL, $1 \mathrm{C} 4 \mathrm{H}, 2 \mathrm{CHS}, 1 \mathrm{~F} 3 \mathrm{H}, 1 \mathrm{~F} 3^{\prime} H, 2 \mathrm{DFR}, 2 \mathrm{LAR}$, and $2 I A A$ (Table 1). Three $P A L, 1 C 4 H, 2 C H S$, and $1 F 3^{\prime} H$ in non-colored area were down-regulated compared with the colored area. Two DFR, $2 L A R$, and $2 I A A$ in non-colored area were up-regulated compared with the colored area (Fig. $7 \mathrm{a})$, involving the entire anthocyanin metabolism pathway key enzyme gene metabolic pathway.

Table 1 Genes related to anthocyanin synthesis in the variegated peanut testa among different comparison groups.

\begin{tabular}{|l|l|l|l|l|l|l|}
\hline gene ID F1-B1 & F1 FPKM & B1 FPKM & FDR & log2FC & regulated & annotation \\
\hline arahy.Tifrunner.gnm1.ann1.0B4MFB & 9.054759 & 1.449418 & 0.000731142 & -2.718222922 & down & PAL \\
\hline arahy.Tifrunner.gnm1.ann1.NB2RRK & 9.630893 & 0.917825 & $8.56 \mathrm{E}-11$ & -3.465122425 & down & PAL \\
\hline arahy.Tifrunner.gnm1.ann1.PUMP6L & 12.603869 & 3.956659 & 0.004951254 & -1.736346256 & down & PAL \\
\hline arahy.Tifrunner.gnm1.ann1.AYA1A5 & 2.3203895 & 0.26419 & 0.00547686 & -3.06931628 & down & C4H \\
\hline arahy.Tifrunner.gnm1.ann1.CY60UM & 22.02194 & 6.7419675 & 0.003171101 & -1.771575358 & down & DHS2 \\
\hline arahy.Tifrunner.gnm1.ann1.6J3HHE & 10.014363 & 0.6830215 & $5.04 \mathrm{E}-10$ & -3.917616027 & down & CHS \\
\hline
\end{tabular}




\begin{tabular}{|c|c|c|c|c|c|c|}
\hline arahy.Tifrunner.gnm1.ann1.UDJX6I & 91.9906315 & 20.076782 & $1.65 \mathrm{E}-05$ & -2.256203498 & down & $\mathrm{CHS}$ \\
\hline arahy.Tifrunner.gnm1.ann1.CB6084 & 0.550337 & 9.0050005 & $4.54 \mathrm{E}-09$ & 3.958349247 & up & IAA14 \\
\hline arahy.Tifrunner.gnm1.ann1.QUY0YV & 0.6811405 & 5.7989815 & 0.000156105 & 3.016455081 & up & IAA14 \\
\hline arahy.Tifrunner.gnm1.ann1.X2EPYD & 0 & 14.942496 & $8.91 \mathrm{E}-43$ & Inf & up & CSNK2B \\
\hline gene ID & F2 FPKM & B2 FPKM & FDR & $\log 2 \mathrm{FC}$ & regulated & annotation \\
\hline arahy.Tifrunner.gnm1.ann1.NB2RRK & 5.903561 & 0.920466 & $8.56 \mathrm{E}-11$ & -3.465122425 & down & PAL \\
\hline arahy.Tifrunner.gnm1.ann1.K8H9R8 & 11.108361 & 26.2737925 & 0.005593953 & 1.202514755 & up & F3'H \\
\hline arahy.Tifrunner.gnm1.ann1.HE8J5U & 27.034563 & 62.0989035 & 0.009349418 & 1.160958266 & up & $\mathrm{F} 3 \mathrm{H}$ \\
\hline arahy.Tifrunner.gnm1.ann1.X8EVF3 & 29.294177 & 152.0960005 & $1.81 \mathrm{E}-05$ & 2.334863116 & up & DFR \\
\hline arahy.Tifrunner.gnm1.ann1.7JZ58T & 11.6955015 & 144.9762875 & $4.15 \mathrm{E}-08$ & 3.60358824 & up & DFR \\
\hline arahy.Tifrunner.gnm1.ann1.M05HM0 & 0.8104485 & 8.8487325 & $8.14 \mathrm{E}-13$ & 3.265464934 & up & LAR \\
\hline arahy.Tifrunner.gnm1.ann1.T1J2UZ & 1.2703215 & 18.3464365 & $2.87 \mathrm{E}-08$ & 3.848946241 & up & LAR \\
\hline arahy.Tifrunner.gnm1.ann1.D1ITL5 & 1.7008935 & 0.1929725 & 0.000903522 & -3.16215485 & down & BG \\
\hline arahy.Tifrunner.gnm1.ann1.13Q8F8 & 30.508549 & 0 & 2.99E-32 & \#NAME? & down & $\mathrm{EC}$ \\
\hline arahy.Tifrunner.gnm1.ann1.49I11A & 12.856979 & 3.036938 & $2.45 \mathrm{E}-07$ & -2.118638966 & down & POD \\
\hline arahy.Tifrunner.gnm1.ann1.D687JL & 0.4383385 & 5.6579165 & $8.33 \mathrm{E}-08$ & 3.520180624 & up & ORR10 X1 \\
\hline arahy.Tifrunner.gnm1.ann1.CB6084 & 0.550337 & 9.0050005 & $4.54 \mathrm{E}-09$ & 3.958349247 & up & IAA14 \\
\hline arahy.Tifrunner.gnm1.ann1.QUY0YV & 0.6811405 & 5.7989815 & 0.000156105 & 3.016455081 & up & IAA14 \\
\hline
\end{tabular}

Analysis of transcription factors differentially expressed in transcriptome. The transcription factors in the sequencing results were analyzed and it was found that 9 differentially expressed MYBs were screened from 410 MYB transcription factors, 4 differentially expressed bHLH were screened from 278 bHLH transcription factors, and no differentially expressed in WD40 family transcription factors(Fig.7b). All MYB regulatory factor genes were enriched in the 2 metabolic pathways of splicing and plant circadian transduction,and all bHLH regulatory factor genes were enriched in the two metabolic pathways of plant hormone signal transduction and plant circadian rhythm.However, the differentially expressed MYB and bHLH transcription factors were not annotated clearly among the metabolic pathways, and the regulatory role of these differential transcription factors in the metabolic pathway was unclear (Table S8). 


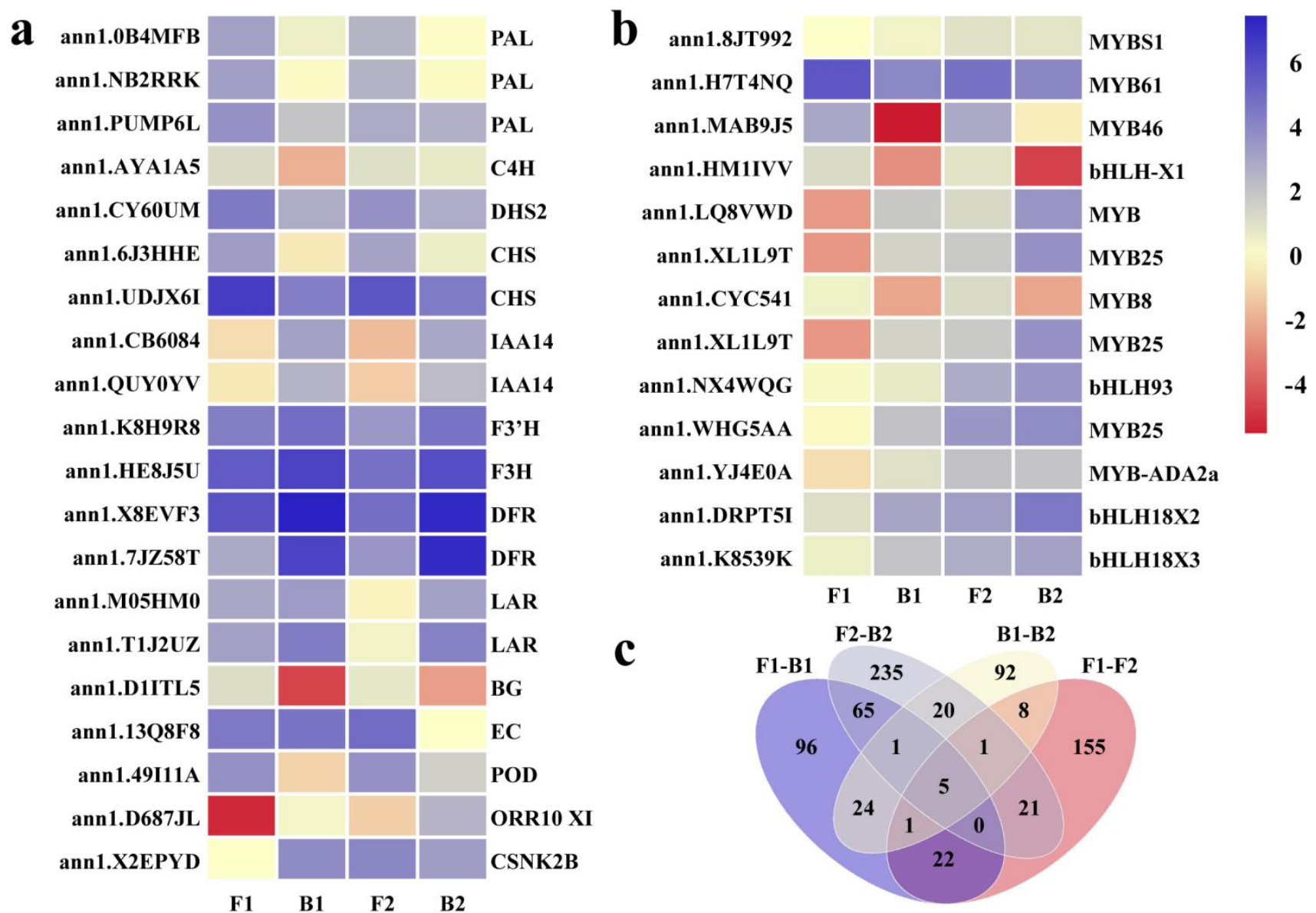

Figure 7.a,b: Heat map of DEGs related to anthocyanin synthesis in the variegated peanut testa among different comparison groups of structural genes and transcription factors, respectively.Gene expression was scaled in this analysis using FPKM Z-scores based on the mean value of two biological replicates in the heatmap. The key is located on the right hand side in each case with FPKM values increasing from blue to orange.c:The Veen diagram of DEGs among different comparison groups.

Joint analysis of transcriptome and metabolome. The combined analysis of metabolome and transcriptome showed that the differential genes in the flavonoid biosynthetic pathway are directly related to the synthesis of delphinidin and cyanidin. The F1-B1 correlation results showed that higher delphinidin and cyanidin content in colored area compared with the non-colored area caused variegated testa, due to the up-regulation of $2 \mathrm{CHS}$ and 1 $\mathrm{C} 4 \mathrm{H}$ in colored area produces. The F2-B2 correlation results showed that the up-regulation of $2 \mathrm{DFRs}, 1 \mathrm{~F} 3^{\prime} H, 1$ $F 3 H$, and $2 L A R s$ in non-colored area caused the accumulation of procyanidin, but leading to the significant increase in the content of delphinidin and cyanidin in colored area compared with the non-colored area (Fig. 8). 


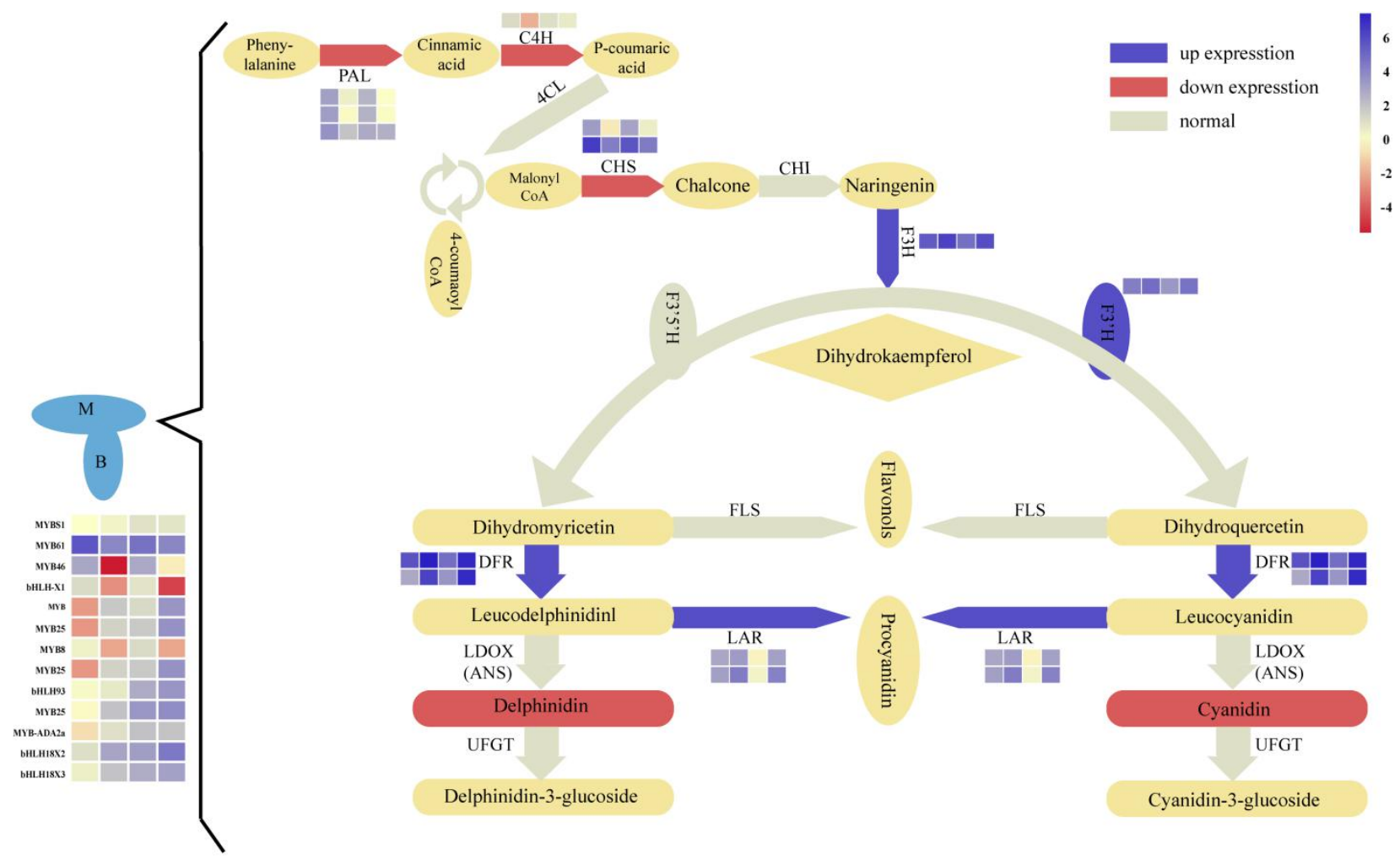

Figure 8. Combined analysis of transcriptome and metabolome of flavonoid biosynthesis.Red graphical represents down-regulation of gene expression, bule graphical represents down-regulation of gene expression, and gray graphical indicate that there is no significant difference in gene expression.

qPCR analysis of differential gene expression levels. Fluorescence quantitative qPCR verification was performed on 20 genes related to anthocyanin metabolism. The results showed that nine genes were verified in F1-B1 (Fig. 9a), F2-B2 (Fig. 9b), B1-B2,(Fig. 9c), and 11 genes were verified in F1-F2 (Fig. 9d), which were consistent with transcriptome results. The 20 selected differential genes in the four comparison groups showed similar qPCR expression trends to the transcriptome detection results (Fig. 9). 

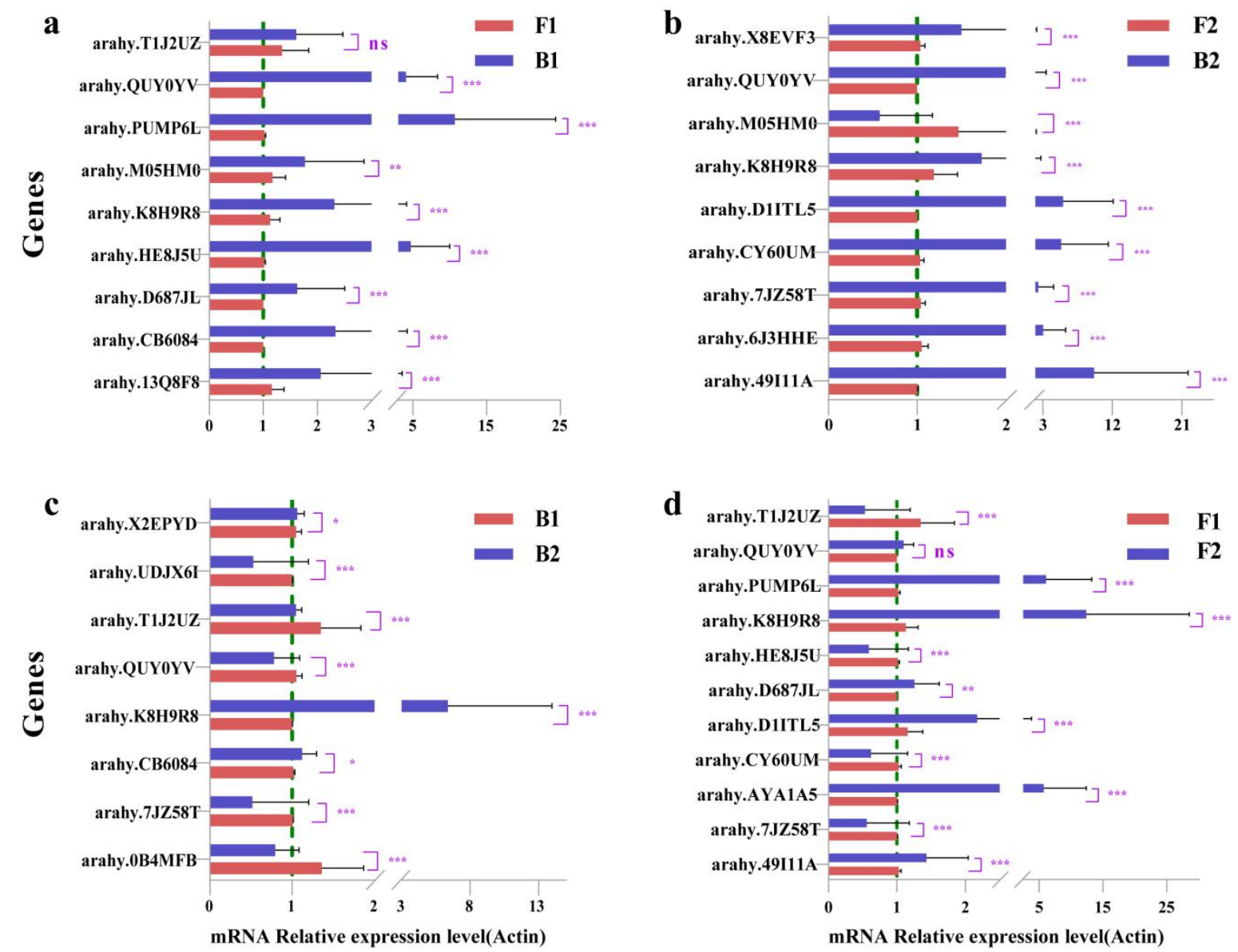

Figure 9. Differential genes qRT-PCR verificated results. a,b,c and d represents F1-B1,F2-B2,B1-B2 and F1-F2, respectively. The $\mathrm{x}$-axis shows the relative gene expression levels analyzed by qRT-PCR. ${ }^{*}, \mathrm{p}<0.05 ;{ }^{* *}, \mathrm{p}<0.01$; and ${ }^{* * *}, \mathrm{p}<0.001$.

\section{Discussion}

Peanut is one of the five largest oil crops in the world, which testa is enriched with biologically active substances. The testa color displays significant differences. Anthocyanin is one of the key substances for color variation in plants ${ }^{34}$. The ingenious sampling is where this research differs from previous studies. In this study, accession VG-01 material with variegated testa was used,and the color differentiation of the testa occurred with the same genetic background, allowing for direct comparison of mechanisms underlying color variation within the same plant,which makes this study worth pondering. At the same time, the sequencing results were compared and analyzed to both diploid wild and tetraploid cultivated peanuts. Moreover, the molecular regulation mechanism of anthocyanin synthesis has not been previously found in the variegated peanut testa, so this research is of significance to the field of research.

CHS of circadian pathway determines the early differentiation of peanut testa color. The synthesis of pigments in the non-colored and colored area is comprehensively regulated by multiple structural genes in the variegated peanut testa. The analysis results showed that two key CHS genes are the first to regulate pigment synthesis. The up-regulated expression of $\mathrm{CHS}$ provides a large amount of substrate for the metabolism of anthocyanins downstream of the colored area of variegated testa. Deng et al. identified $10 \mathrm{CHS}$ that were significantly up-regulated,,which used materials of different colors and different parts on the same wild banana pee ${ }^{35}$. Gao et al. adopted transcriptome sequencing to screen RsCHS- B2 and found that RsCHS is up-regulated in red radishes when they formed fleshy roots, which were important genes for red color ${ }^{36}$. Wan et al. studied the increase in $C H S$ expression during the growth process from DAF20d to 
DAF60d, which promoted the color accumulation in the peanut testa ${ }^{24}$. The significant difference in our study was that two $C H S$ enriched in plant circadian metabolic pathways were identified. We hypothesize that the two CHS may be key genes for the color differentiation between the peanut mottling and non-colored areas in the early testa development. The up-regulation of the two CHS increases the cyanidin content in the red area of the variegated testa.

\section{Up-regulated genes in the flavonoid metabolism pathway expressing as pre-products accumulated in colored area.} In the flavonoid synthesis pathway at DAF30, three $P A L$, one $C 4 H$, and two $C H S$ genes are all up-regulated in colored area, which provides a preliminary product for pigment accumulation in colored area. Wan et al. analyzed the pink peanut testa ZH16 and the results showed that the expression of PAL, 4CL, CHS, CHI, and other enzymes increased with the peanut testa growth ${ }^{24}$. Previous studies reported that $F 3 H, F 3^{\prime} H$, and $F 3^{\prime} 5^{\prime} H$ could catalyze the synthesis of three dihydroflavonols (DHK, DHQ, and DHM), where DHK eventually produced brick red geranium pigment, DHQ produced red cyanidin glycosides, and DHM produced blue to purple delphinidin glycosides ${ }^{37}$. In the process of flower color synthesis in dahlia, the expression of $F 3 H$ is significantly down-regulated, changing the color from orange to light red, or even white ${ }^{38}$. Interestingly, we identified the up-regulation of $F 3 H$ and $F 3^{\prime} H$ in non-colored area at DAF45. This may be due to the up-regulation of downstream genes in the flavonoid metabolism pathway and that the reverse regulation promotes the increase of $F 3 H$ and $F 3^{\prime} H$ expression. Previous studies have shown that silencing the endogenous gene $F 3$ ' $H$ with RNA interference technology could increase the delphinidin content and make the petals of chrysanthemums appear a new type of blue ${ }^{39}$. Therefore, the pigment accumulation pattern of variegated peanut testa may be similar to the studies citeed above.

Procyanidin accumulating in the later stage of variegated peanut testa. $D F R$ is the first key downstream gene that affects the anthocyanin synthesis branch in the variegated testa. High expression of $D F R$ is advantageous to the accumulation of plant anthocyanins. For example, brick red petunidin 3-O-glucoside can be obtained if DFR is introduced into white gerbera ${ }^{40,41}$. Zhang et al. studied the up-regulation of DFR gene expression in purple leaves in mustard, which contributes to the accumulation of anthocyanins ${ }^{34}$. Different from previous studies, the expression levels of two $D F R$ selected by the transcriptome in our study were higher in non-colored area than in colored area. The metabolomic analysis determined that the content of Delphinidin and Cyanidin in colored area was significantly higher than that in non-colored area. There are two possible explanations for the up-regulation of $D F R$ in non-colored area. One is that there is no significant correlation between DFR enzyme activity and testa anthocyanin accumulation during pigment synthesis. Wang et al. have reported that DFR enzyme activity in the pericarp was not closely related to anthocyanin synthesis in litchi ${ }^{42}$. Furthermore, there is no significant correlation detected between anthocyanin content and DFR enzyme activity in sweet cherry ${ }^{43}$. The second reason for the significant up-regulation of $D F R$ expression in non-colored area may be that the up-regulated expression of $L A R$ causes the leuco anthocyanins to accumulate to procyanidin in the testa. The leuco anthocyanidins are synthesized and accumulated in vacuoles under the catalysis of $\mathrm{LAR}^{44}$.

Hormone inhibition of pigment accumulation in colored area of variegated peanut testa. Hormones have an antagonistic effect on the synthesis of pigments. The transcription factors bHLH and MYB indirectly regulate the synthesis of anthocyanins by regulating the synthesis of hormones. The results showed that 4 differentially expressed bHLH and 9 MYB regulatory genes were not clearly annotated in the metabolic pathway. It is speculated that bHLH and MYB regulatory genes in the plant circadian rhythm pathway may promote the synthesis of CHS to facilitate the accumulation of metabolites in the early stage and make the colored area darker. The synthesis of testa pigment was indirectly regulated by hormones in the plant signal transduction pathway. Wan et al. identified 16 DEGs related to plant hormone signal transduction, four of which were down-regulated by the AUX signal and concluded that hormones had an 
antagonistic effect on pigment synthesis ${ }^{25}$. In this study, we found that the expression levels of 2 IAA14 hormone-regulated genes in non-colored area at DAF30 and DAF45 were all up-regulated, which is consistent with previous studies.

Joint analysis and mutual verification of pigment accumulation. The results of the combined analysis of the transcriptome and metabolome data were corroborated. We found that the metabolic pathway was the most closely integrated pathway of flavonoid biosynthesis, and 9 most closely integrated genes are all key genes in the anthocyanin metabolic pathway. This suggested that the selected $2 C H S, 1 C 4 H, 2 D F R, 1 F 3^{\prime} H, 1 F 3 H$, and 2 LAR were the key genes for anthocyanin synthesis in peanut. Two different metabolites, Delphinidin and Cyanidin, were enriched in this pathway. The comparison results demonstrated that Delphinidin and Cyanidin are the primary pigments that make the colored area darker than the non-colored area. The transcriptome and metabolome of the colored and non-colored of variegated testa are closely integrated.Following analysis, it was determined that delphinidin and cyanidin were the key pigments that make the colored area different from the non-colored area in the variegated testa.

In summary, DEGs in this study were involved in 96 metabolic pathways. Phenylalanine metabolism, phenylpropanol biosynthesis, flavonoids and flavonol biosynthesis, flavonoid biosynthesis, plant hormone signal transduction, and circadian rhythm plants were related pigment synthesis in variegated testa. During the testa development, 14 main structural enzyme genes and 13 regulatory genes affected the pigment synthesis, including phenylalanine ammonia lyase, cinnamic 4-hydroxylase enzyme, chalcone synthase, dihydroflavonol-3- hydrogenase, dihydroflavonol-3'-hydrogenase, dioxyflavonol-4-reductase, leuco anthocyanins, 4 bHLHs, and 9 MYBs regulatory genes.The results in this study reveal for the molecular regulation mechanism of pigment synthesis in peanut testa, which is of great significance to the development of functional genomics in variegated peanut.

\section{Materials and methods}

\section{Materials}

The variegated testa (accession VG-01) is composed of two parts, the non-colored area (white) and the colored area (red). The seeds were provided by the Peanut Breeding Laboratory of Hebei Agricultural University in P.R.China.VG-01 was flowering and needling on May 6, 2018, and was marked by the hanging thread method at day after flowering and needling(DAF)30, 35, 40, 45, 50, and 55 days. The variegated seeds testa used for RNA extraction and repeated twice.

Characterization by stereomicroscope and chromatic aberration value. The peanut testa after needling for different days was sliced and the color changes were observed using the stereomicroscope (OLYMPUS,SZ61). Different testa parts at different periods were taken, and "L", "a", and "b" values were measured using a colorimeter (Konica Minolta Colorimeter CR-10Plus), where "L" represents the lightness value, "a" represents red and green values, and "b" represents yellow and blue value. F1 and F2 represent the sample names of colored area at DAF30 and DAF45,respectively. B1 and B2 represent the sample names of non-colored area at DAF30 and DAF45, respectively.

Anthocyanins extraction from variegated testa. Peanut testa in different colored areas at DAF30 and DAF45 was stored at $-80{ }^{\circ} \mathrm{C}$. Samples were crushed using a mixer mill (MM 400, Retsch) with a zirconia bead (1.5 min, $30 \mathrm{~Hz}$ ). $100 \mathrm{mg}$ powder was weighted and extracted overnight at $4{ }^{\circ} \mathrm{C}$ with $1.0 \mathrm{~mL} 70 \%$ aqueous methanol. Following centrifugation at 10,000g for $10 \mathrm{~min}$, the extracts were absorbed (CNWBOND Carbon-GCB SPE Cartridge, 250mg, $3 \mathrm{~mL}$; ANPEL, Shanghai, China, www.anpel.com.cn/cnw) and filtrated (SCAA-104,0.22 $\mu \mathrm{m}$ pore size; ANPEL, Shanghai, China, http://www.anpel.com.cn/) before Liquid chromatography tandem mass spectrometry (LC-MS) analysis. 
Species and quantitative analysis of anthocyanin in variegated peanut testa. LC-MS/MS was adopted to qualitatively and quantitatively detect the different areas of the variegated testa. The testa was dissolved in dimethyl sulfoxide or methanol and stored at $-20{ }^{\circ} \mathrm{C}$, which was diluted with $70 \%$ methanol to different gradient concentrations before mass spectrometry analysisUltra-high-performance liquid chromatography and tandem mass spectrometry (MS/MS) (Applied Biosystems 4500 QTRAP, http://www.appliedbiosystems.com.cn) was adopted to collect data. Self-built database MWDB (metware database), metabolite synthesis public database MassBank (http://www.massbank.Jp/), KNAPSAcK(http://kanaya. Naist. jp/ KNApSAcK/) HMDB (http://www. hmdb.Ca/) (Wishart et al.2013) ${ }^{45}$, MoToBD (http://www. ab.wur.nl/moto/) and METLIN (http://metlin. scripps. edu/ index.php) (Zhu et al.2013) ${ }^{46}$ were used to qualitatively analyze the primary and secondary spectral data detected by mass spectrometry.Analyst 1.6.3 software was used to analyze the detected data. MultiaQuant software was used to integrate and calibrate the mass spectrometry results to finally obtain the different metabolites.

Transcriptome sequencing and DEGs analysis. The samples were sequenced and analyzed using the Illumina sequencing platform. The original data sequencing results were compared and analyzed with the diploid wild (Araip.K30076.a1.m1\&\&Aradu.V14167.a1.M1,Peanutbase) and Tifrunner genomes(http://peanutbase.org/datahypogaea/ Tifrunner.gnm1.ann1.CCJH/) and functional annotations were performed. The Fragments per kilobase of transcript sequence per million base pairs (FPKM) values in four samples were calculated and DEseq software was used to perform DEGs analysis. Fold Change $\geqslant 2$ and FDR $<0.01$ were chosen as DEGs screening criteria. The Benjamini-Hochberg was used to correct the significance $p$-value obtained from the original hypothesis test to reduce the false positive rate, and FDR was used as the key indicator for DEGs screening.

GO and KEGG analysis of DEGs. The Gene ontology(GO) and Kyoto Encyclopedia of Genes and Genomes(KEGG) pathway enrichment analysis of the DEGs was based on the standard of p-value $<0.05$ and FDR $\leqslant 0.05$. The GO predicted protein information was obtained when the similarity $>30 \%$, E-value $<$ Ie- 5 using the BLAST comparison tool. The metabolic synthesis pathway was annotated by KEGG, which was obtained when compared with the KEGG database under the condition of E-value $<$ le3.

Joint analysis of transcriptome and metabolome. The differential genes and metabolites screened from the metabolome and transcriptome were jointly analyzed according to the Pearson correlation analysis method. The joint analysis was carried out on the metabolome and transcriptome data based on the standard of the correlation coefficient threshold $\geqslant 0.8$ and correlation $\mathrm{P}<0.05$.

Verification by qRT-PCR. The selected 20 DEGs with the colored area were all verified by real-time quantitative PCR (qRT-PCR). Total RNA was extracted by the TRIZOL method, and the corresponding cDNA was synthesized. According to the sequence library required for sequencing, the fluorescent real-time quantitative PCR primers were designed (Table S9) by using Primer Premier5.0 software, in which the peanut actin ACT7 gene was used as the internal reference gene. The $\Delta \Delta \mathrm{Ct}$ data analysis method was used for gene expression analysis and $2^{-\Delta \Delta \mathrm{Ct}}$ was used to calculate the relative gene expression $^{47}$. The expression level in the target gene of the control part (B1, B2 non-colored areas) was 1, and two replicates were set in this experiment.

\section{References}

1. Wang, Y. et al. Research progress of peanut aflatoxin control and detection methods. Liaoning Agricultural Sciences. 05, 66-68(2019).

2. Bulgakov, V. P., Avramenko, T. V. \& Tsitsiashvili, G. S. Critical analysis of protein signaling networks involved in the regulation of plant secondary metabolism: focus on anthocyanins. Crit Rev Biotechnol. 37, 685-700(2017). 
3. Wang, S. Molecular physiological mechanism of anthocyanin accumulation in purple sweet potato tubers. Fujian Agriculture and Forestry University (2016).

4. Chen, D. et al. Alternatively Spliced BnaPAP2.A7 Isoforms Play Opposing Roles in Anthocyanin Biosynthesis of Brassica napus L. Front Plant Sci. 11, 983(2020).

5. Sun, Q. et al. Color change and pigment deposition of colored peanut testa. Acta Arachis. 44, 1-6(2015).

6. $\mathrm{Hu}$, J. et al. Ultraviolet B-induced MdWRKY72 expression promotes anthocyanin synthesis in apple. Plant Sci. 292, 110377(2020).

7. Fukusaki, E. et al. Flower color modulations of Torenia hybrida by downregulation of chalcone synthase genes with RNA interference. J Biotechnol. 111, 229-40(2004).

8. Chen, Q. et al. Research progress of MYB transcription factors in plants. Genomics and Applied Biology. 28, 365-372(2009).

9. van der Krol, A. R., Mur, L. A., de Lange, P., Mol, J. N. \& Stuitje, A. R. Inhibition of flower pigmentation by antisense CHS genes: promoter and minimal sequence requirements for the antisense effect. Plant Mol Biol. 14, 457-66(1990).

10. Yamazaki, M. et al. Metabolomics and differential gene expression in anthocyanin chemo-varietal forms of Perilla frutescens. Phytochemistry. 62, 987-95(2003).

11. Zhu, Z. \& Lu, Y. Anthocyanin metabolic pathway and plant color variation. Acta Botanica Sinica. 51, 107-119(2016).

12. Zhang, X. Regulation of ANS, ANR and LAR genes in anthocyanin metabolism of Strawberry. Sichuan Agricultural University (2013).

13. Chen, Y. Cloning and functional verification of CsUGGT gene from tea plant. Hunan Agricultural University (2017).

14. Elomaa, P. et al. Activation of anthocyanin biosynthesis in Gerbera hybrida (Asteraceae) suggests conserved protein-protein and protein-promoter interactions between the anciently diverged monocots and eudicots. Plant Physiol. 133, 1831-42(2003).

15. Baudry, A. et al. TT2, TT8, and TTG1 synergistically specify the expression of BANYULS and proanthocyanidin biosynthesis in Arabidopsis thaliana. Plant J. 39, 366-80(2004).

16. Almeida, J., Carpenter, R., Robbins, T. P., Martin, C. \& Coen, E. S. Genetic interactions underlying flower color patterns in Antirrhinum majus. Genes Dev. 3, 1758-67(1989).

17. Xiang, L. L. et al. A Novel bHLH Transcription Factor Involved in Regulating Anthocyanin Biosynthesis in Chrysanthemums (Chrysanthemum morifolium Ramat.). PLoS One. 10, e0143892(2015).

18. Xia, H. et al. Comparative transcriptome analysis of anthocyanin synthesis in black and pink peanut. Plant Signal Behav. 15, 1721044(2020).

19. Shang, X. et al. Advances in molecular mechanism of plant spot synthesis. Journal of Horticulture. 41, 1485-1494(2014).

20. Koseki, M., Goto, K., Masuta, C. \& Kanazawa, A. The star-type color pattern in Petunia hybrida 'red Star' flowers is induced by sequence-specific degradation of chalcone synthase RNA. Plant Cell Physiol. 46, 1879-83(2005).

21. Lin, W. The star-type color pattern in Petunia hybrida 'Red' Star flowers is induced by sequence-specific degradation of chalcone synthase RNA. Plant \& Cell Physiology (2017).

22. Chiou, C. Y. \& Yeh, K. W. Differential expression of MYB gene (OgMYB1) determines color patterning in floral tissue of Oncidium Gower Ramsey. Plant Mol Biol. 66, 379-88(2008).

23. Li, H., Qiu, J., Sun, X. \& Liang, X. Cloning and sequence analysis of genes related to anthocyanin synthesis in Peanut Cultivars with different testa colors. Journal of Tropical Crops. 39, 600-605(2017).

24. Wan, L. et al. Transcriptomic profiling reveals pigment regulation during peanut testa development. Plant Physiol Biochem. 125, 116-125(2018). 
25. Li, M. et al. Cloning and expression analysis of peanut Flavanone-3-hydroxylase Gene ahf3h. Shandong Agricultural Sciences. 45, 1-6(2013).

26. Zhang, Y. L., Fang, Z. Z., Ye, X. F. \& Pan, S. L. Identification of candidate genes involved in anthocyanin accumulation in the peel of jaboticaba (Myrciaria cauliflora) fruits by transcriptomic analysis. Gene. 676, 202-213(2018).

27. Liu, T. et al. Transcriptome analyses reveal key genes involved in skin color changes of 'Xinlimei' radish taproot. Plant Physiol Biochem. 139, 528-539(2019).

28. Jia, Z. et al. Transcriptome and anthocyanin accumulation mechanism in fruit synthesis of 'Hongyang' Kiwifruit. in School of Chinese Academy of Sciences (Wuhan Botanical Garden).(2017).

29. Jin, X. Synthesis of anthocyanin from Chrysanthemum morifolium based on high throughput sequencing. Beijing Forestry University (2013).

30. Jin, X., Huang, H., Wang, L., Sun, Y. \& Dai, S. Transcriptomics and Metabolite Analysis Reveals the Molecular Mechanism of Anthocyanin Biosynthesis Branch Pathway in Different Senecio cruentus Cultivars. Front Plant Sci. 7 , 1307(2016).

31. Bai, Y., Dougherty, L. \& Xu, K. Towards an improved apple reference transcriptome using RNA-seq. Mol Genet Genomics. 289, 427-38(2014).

32. Venturini, L. et al. De novo transcriptome characterization of Vitis vinifera cv. Corvina unveils varietal diversity. BMC Genomics. 14, 41(2013).

33. Li, Y. et al. MicroRNA858 negatively regulates anthocyanin biosynthesis by repressing AaMYBC1 expression in kiwifruit (Actinidia arguta). Plant Sci. 296, 110476(2020).

34. Zhang, D. et al. Genome-wide identification and expression analysis of anthocyanin biosynthetic genes in Brassica juncea. Journal of Integrative Agriculture. 19, 1250-1260(2020).

35. Deng, S. Study on the molecular mechanism of color difference synthesis of wild banana peel based on RNA seq. Fujian Agriculture and Forestry University (2018).

36. Gao, J., Li, W. B., Liu, H. F. \& Chen, F. B. De novo transcriptome sequencing of radish (Raphanus sativus L.) fleshy roots: analysis of major genes involved in the anthocyanin synthesis pathway. BMC Mol Cell Biol. 20, 45(2019).

37. Ohno, S., Hori, W., Hosokawa, M., Tatsuzawa, F. \& Doi, M. Post-transcriptional silencing of chalcone synthase is involved in phenotypic lability in petals and leaves of bicolor dahlia (Dahlia variabilis) 'Yuino'. Planta. 247, 413-428(2018).

38. Enrique, G. A. et al. Elicitation with Bacillus QV15 reveals a pivotal role of F3H on flavonoid metabolism improving adaptation to biotic stress in blackberry. PloS one. 15,e0232626 (2020).

39. Brugliera, F. et al. Violet/blue chrysanthemums--metabolic engineering of the anthocyanin biosynthetic pathway results in novel petal colors. Plant Cell Physiol. 54, 1696-710(2013).

40. Li, Y., Gao, Z., Zhang, C., Li, N. \& Liu, C. Research progress of molecular regulation mechanism in anthocyanin synthesis pathway. Journal of ecology. 34, 2937-2942(2015).

41. Johnson, E. T. et al. Alteration of a single amino acid changes the substrate specificity of dihydroflavonol 4-reductase. Plant J. 25, 325-33(2001).

42. Wang, H., Huang, X., Hu, G. \& Huang, H. Study on the relationship between anthocyanin synthesis and related enzymes in Litchi Pericarp. Agricultural Science in China. 2028-2032(2004).

43. Shen, Y. Study on nutritional components and anthocyanins of cherry fruit. Nanjing Forestry University (2014).

44. Gargouri, M., Gallois, B. \& Chaudière, J. Binding-equilibrium and kinetic studies of anthocyanidin reductase from Vitis vinifera. Arch Biochem Biophys. 491, 61-8(2009). 
45. Wishart, D. S. et al. HMDB 3.0--The Human Metabolome Database in 2013. Nucleic Acids Res. 41, D801-7(2013).

46. Zhu, Z. J. et al. Liquid chromatography quadrupole time-of-flight mass spectrometry characterization of metabolites guided by the METLIN database. Nat Protoc. 8, 451-60(2013).

47. Livak, K. J. \& Schmittgen, T. D. Analysis of relative gene expression data using real-time quantitative PCR and the 2(-Delta Delta C(T)) Method. Methods. 25, 402-8(2001).

\section{Acknowledgements}

We thank Key project of science and technology research in colleges and universities of the department of education in Hebei Province (ZD2019051) and Key project of science and technology research of modern seed industry of the department of S\&T in Hebei Province(19226363D) for providing funds.This research was supported by North China Key Laboratory for Crop Germplasm Resources of Education Ministry, College of Agronomy, Hebei Agricultural University and Hebei Yiyuan Ecological Agriculture Technology Co, Ltd.

\section{Author Contributions}

G.M. the corresponding author; G.M. and L.L. developed the concept, planned, coordinated and executed the research. Me.H. performed most of the experiments, analyzed the data, and prepared the Figures and tables; Me.H. wrote the manuscript; J.L., Mi.H. and X.L. performed some analyses and prepared Fig. 1-9; S.C., X.Y. and X.J. supervised the experiments; G.M., Mi.H. and X.Y. modifed the manuscript. All authors read and approved the final manuscript.

\section{Competing interests}

The authors declare no competing interests. 


\section{Figures}

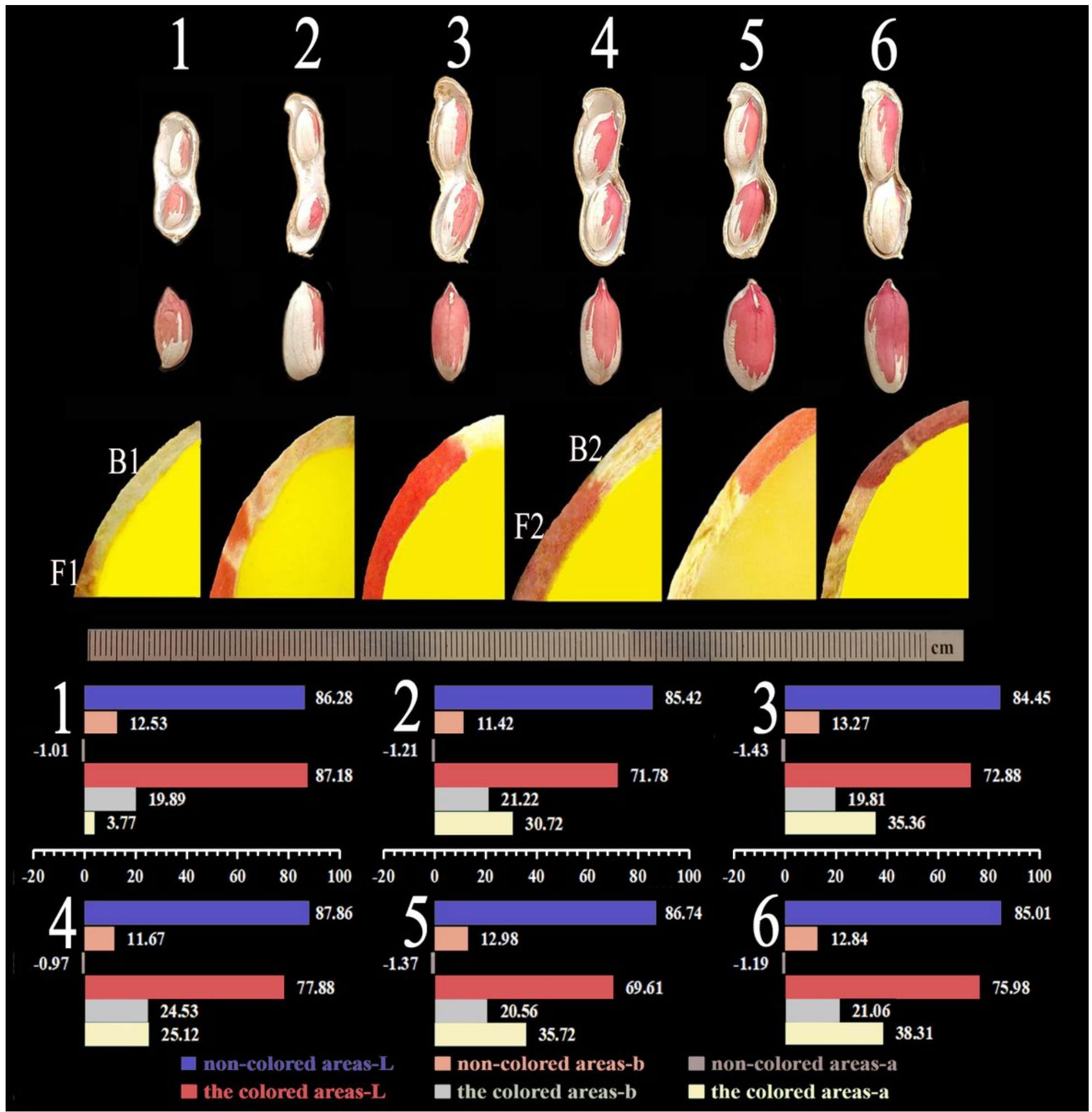

\section{Figure 1}

Phenotype and chromatic aberration changes in the color synthesis of variegated peanut testa. 

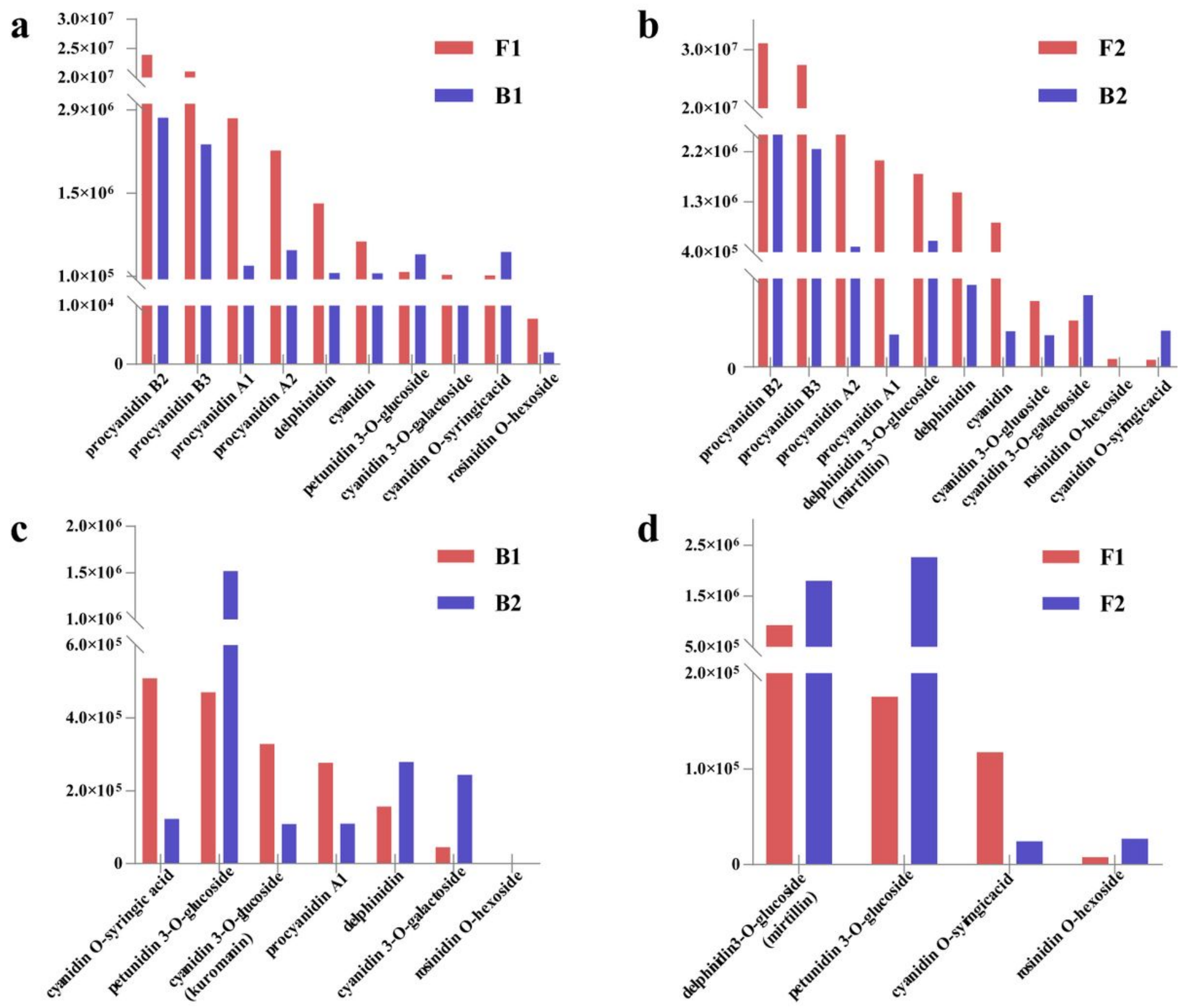

Figure 2

Types and relative content of anthocyanins in different areas of peanut testa at different developmental stages 

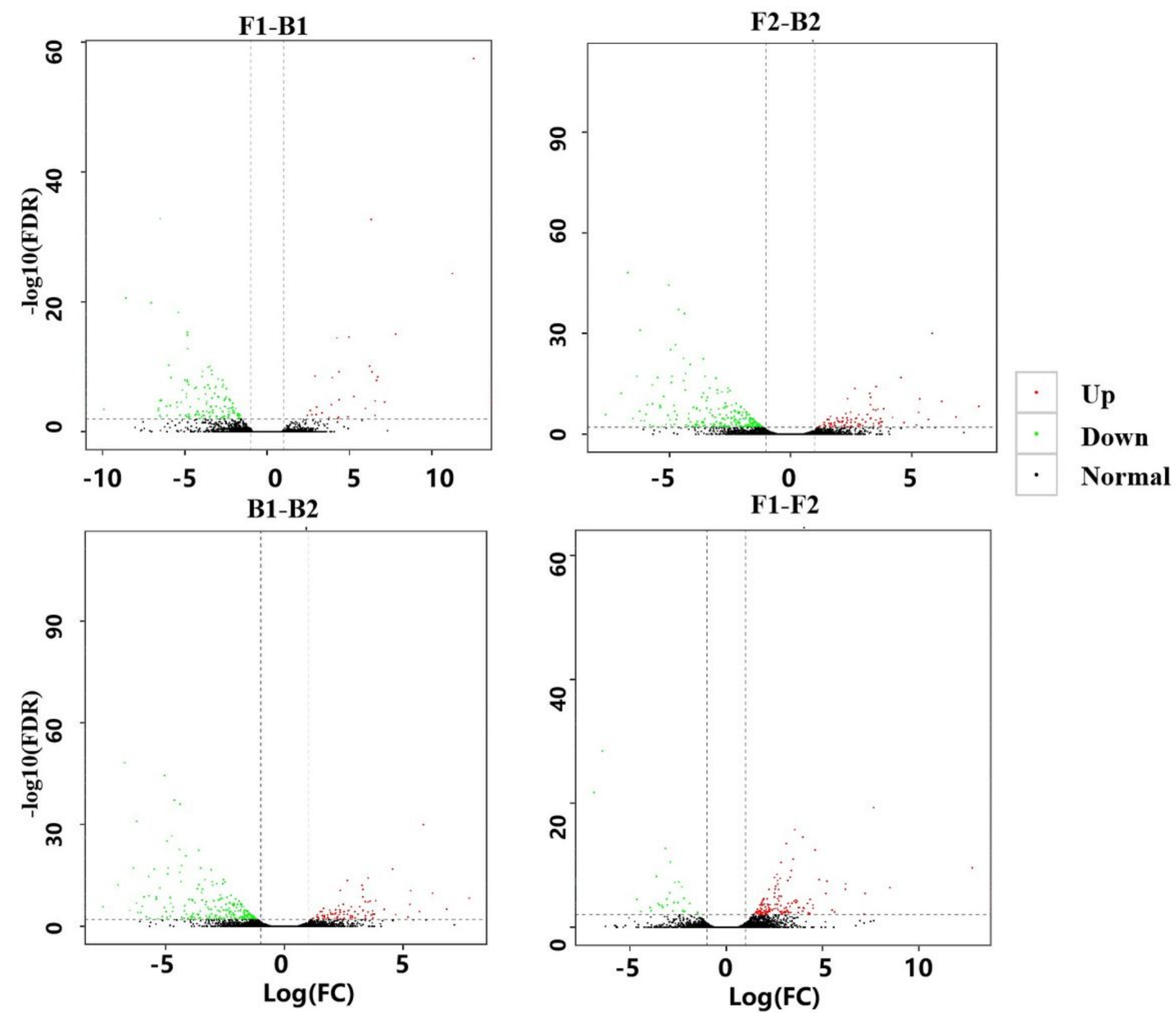

Figure 3

Analysis of the volcano map of different genes in various parts of the peanut testa at different developmental stages 

F1_vs_B1
F2_vs_B2
B1_vs_B2
F1_vs_F2

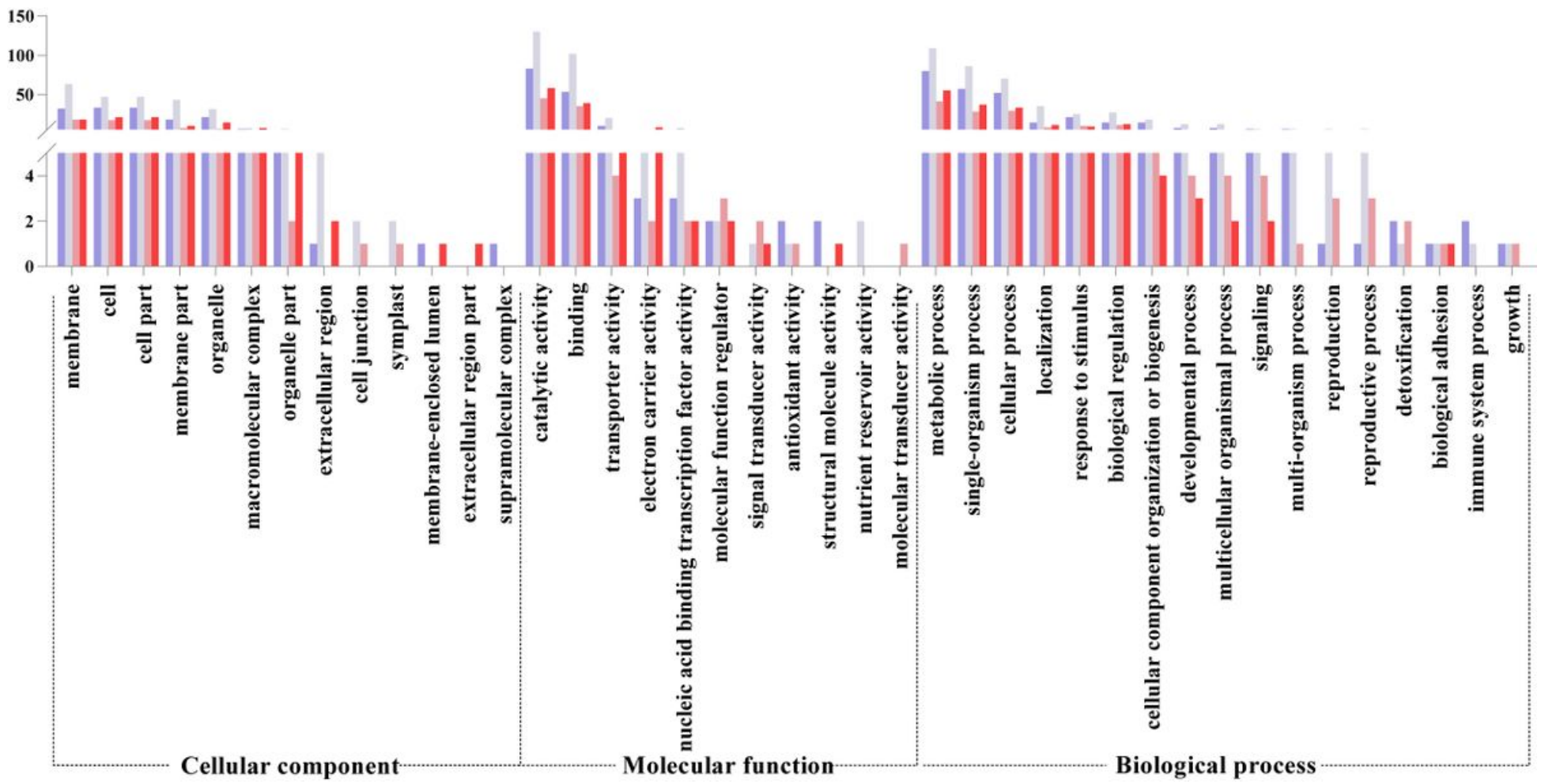

Figure 4

GO functional enrichment classification of variegated peanut testa transcriptome

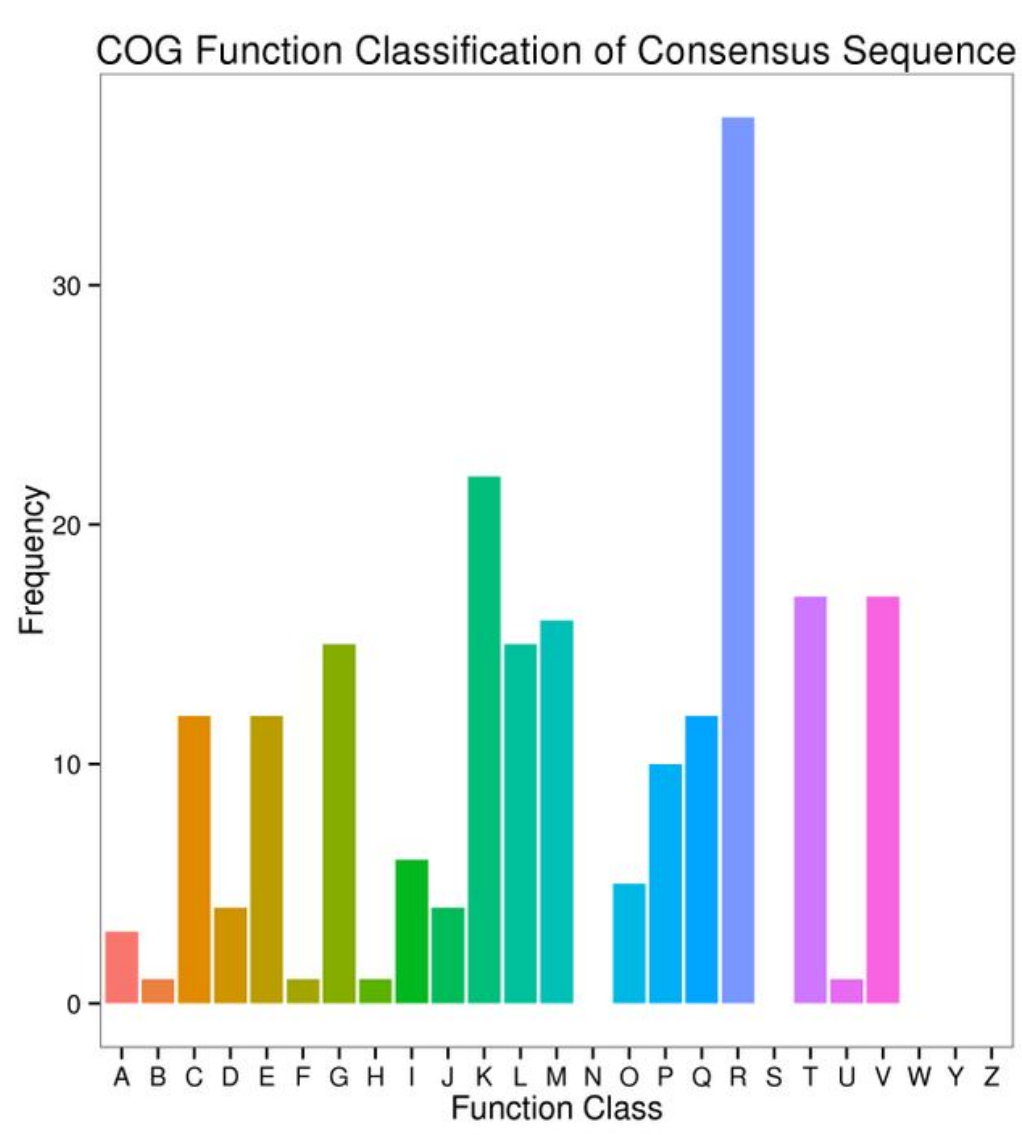

A: RNA processing and modification

B: Chromatin structure and dynamics

C: Energy production and conversion

D: Cell cycle control, cell division, chromosome partitioning

E: Amino acid transport and metabolism

F: Nucleotide transport and metabolism

G: Carbohydrate transport and metabolism

$\mathrm{H}$ : Coenzyme transport and metabolism

I: Lipid transport and metabolism

$\mathrm{J}$ : Translation, ribosomal structure and biogenesis

K: Transcription

L: Replication, recombination and repair

M: Cell wall/membrane/envelope biogenesis

$\mathrm{N}$ : Cell motility

O: Posttranslational modification, protein turnover, chaperones

$\mathrm{P}$ : Inorganic ion transport and metabolism

Q: Secondary metabolites biosynthesis, transport and catabolism

$\mathrm{R}$ : General function prediction only

S: Function unknown

$\mathrm{T}$ : Signal transduction mechanisms

U: Intracellular trafficking, secretion, and vesicular transport

$\mathrm{V}$ : Defense mechanisms

W: Extracellular structures

Y: Nuclear structure

Z: Cytoskeleton 
Figure 5

COG functional classification compared with the tetraploid peanut reference genome

\section{Statistic of Pathway Enrichment}

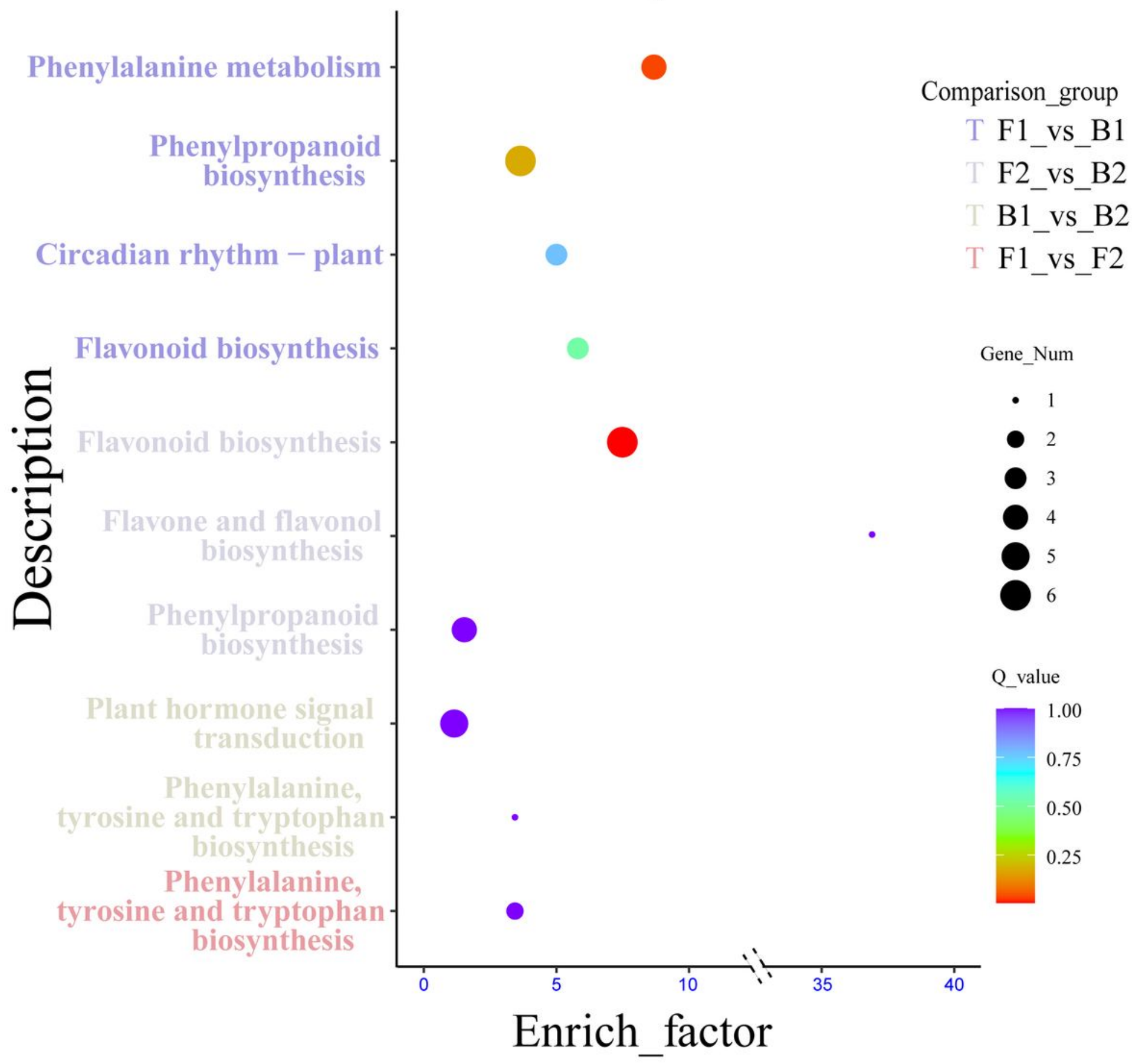

Figure 6

Pathway enrichment analysis of DEGs 


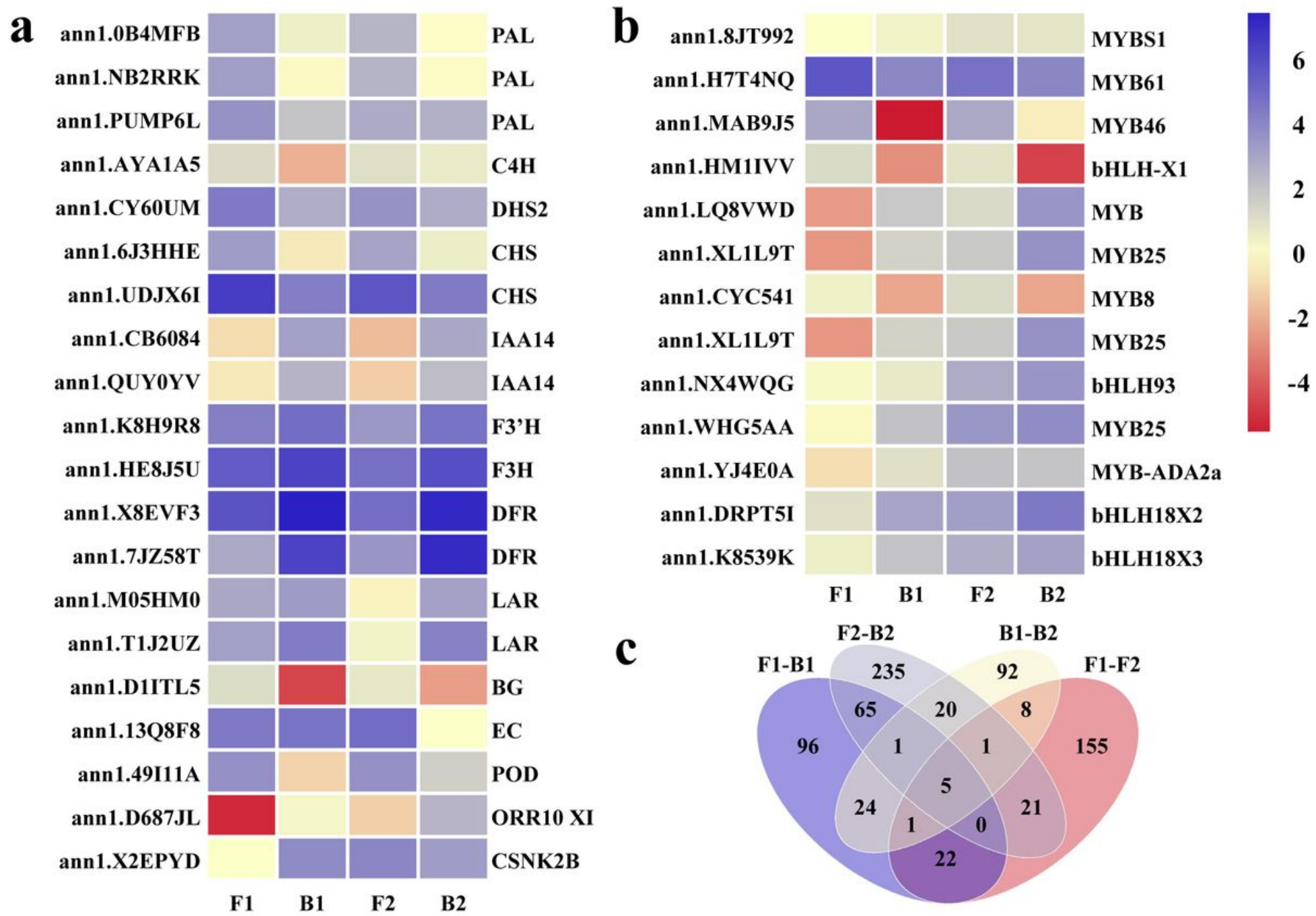

\section{Figure 7}

Heat map of DEGs related to anthocyanin synthesis in the variegated peanut testa among different comparison groups of structural genes and transcription factors, respectively 


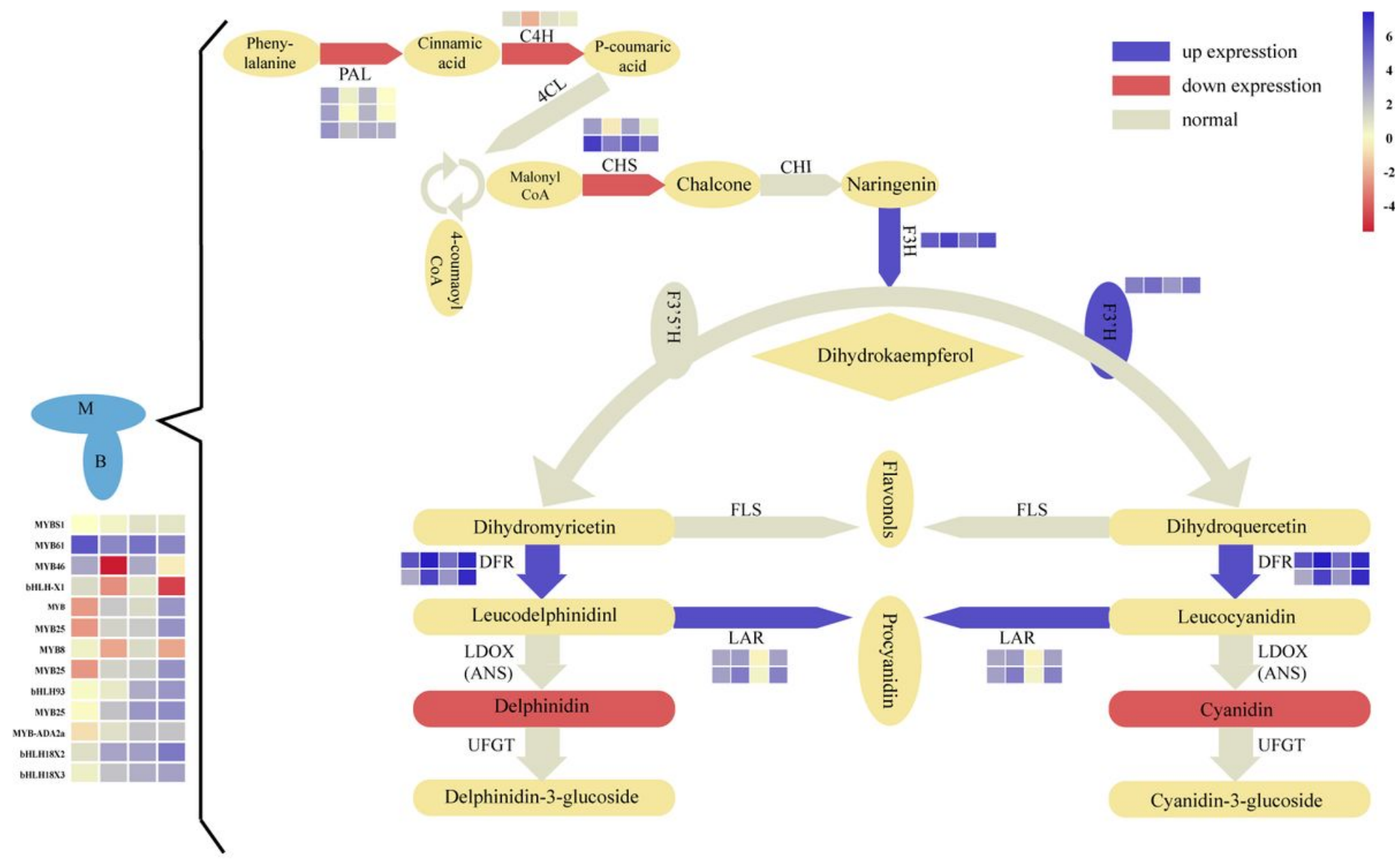

Figure 8

Combined analysis of transcriptome and metabolome of flavonoid biosynthesis 

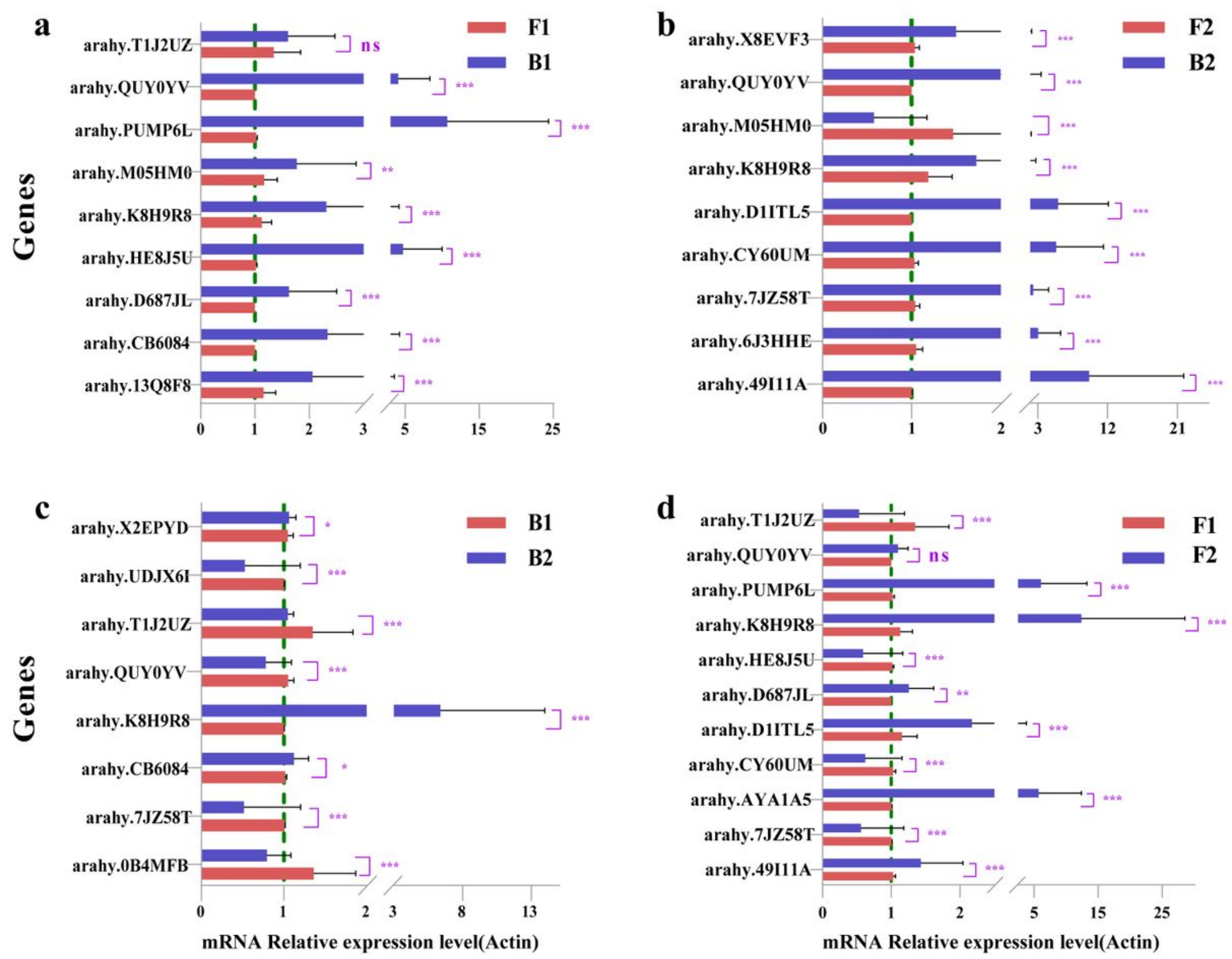

Figure 9

Differential genes qRT-PCR verificated results

\section{Supplementary Files}

This is a list of supplementary files associated with this preprint. Click to download.

- SupplementaryTableS1.xlsx

- SupplementaryTableS2.xlsx

- SupplementaryTableS3.xlsx

- SupplementaryTableS4.xlsx

- SupplementaryTableS5.xlsx

- SupplementaryTableS6.xlsx 
- SupplementaryTableS7.xlsx

- SupplementaryTableS8.xlsx

- SupplementaryTableS9.xIsx 